\title{
вM] Global Health Surgical site infection after gastrointestinal surgery in children: an international, multicentre, prospective cohort study
}

\author{
GlobalSurg Collaborative
}

To cite: GlobalSurg

Collaborative. Surgical site infection after gastrointestinal surgery in children: an international, multicentre, prospective cohort study. BMJ Global Health 2020;5:e003429. doi:10.1136/ bmjgh-2020-003429

\section{Handling editor Senjuti Saha}

- Additional material is published online only. To view, please visit the journal online (http://dx.doi.org/10.1136/ bmjgh-2020-003429).

Received 13 July 2020 Revised 19 August 2020 Accepted 22 August 2020
D Check for updates

(c) Author(s) (or their employer(s)) 2020. Re-use permitted under CC BY. Published by BMJ.

Department of Clinical Surgery, University of Edinburgh

Division of Clinical and Surgical Sciences, Edinburgh, UK

Correspondence to

Ewen M Harrison;

ewen.harrison@ed.ac.uk

\section{ABSTRACT}

Introduction Surgical site infection (SSI) is one of the most common healthcare-associated infections (HAls). However, there is a lack of data available about SSI in children worldwide, especially from low-income and middle-income countries. This study aimed to estimate the incidence of SSI in children and associations between SSI and morbidity across human development settings. Methods A multicentre, international, prospective, validated cohort study of children aged under 16 years undergoing clean-contaminated, contaminated or dirty gastrointestinal surgery. Any hospital in the world providing paediatric surgery was eligible to contribute data between January and July 2016. The primary outcome was the incidence of SSI by 30 days. Relationships between explanatory variables and SSI were examined using multilevel logistic regression. Countries were stratified into high development, middle development and low development groups using the United Nations Human Development Index (HDI).

Results Of 1159 children across 181 hospitals in 51 countries, $523(45 \cdot 1 \%)$ children were from high HDI, 397 (34.2\%) from middle HDI and 239 (20.6\%) from low HDI countries. The 30 -day SSI rate was $6.3 \%(33 / 523)$ in high $\mathrm{HDI}, 12 \cdot 8 \%(51 / 397)$ in middle $\mathrm{HDI}$ and $24 \cdot 7 \%$ (59/239) in low HDI countries. SSI was associated with higher incidence of 30-day mortality, intervention, organ-space infection and other HAls, with the highest rates seen in low $\mathrm{HDI}$ countries. Median length of stay in patients who had an SSI was longer (7.0 days), compared with 3.0 days in patients who did not have an SSI. Use of laparoscopy was associated with significantly lower SSI rates, even after accounting for HDI.

Conclusion The odds of SSI in children is nearly four times greater in low HDI compared with high HDI countries. Policies to reduce SSI should be prioritised as part of the wider global agenda.

\section{INTRODUCTION}

Surgical site infection (SSI) is one of the most common healthcare-associated infections (HAIs) following gastrointestinal surgery. In children, SSI has important social and economic consequences, leading to time away

\section{Key questions}

What is already known?

- There is a lack of data describing the incidence and risk factors for surgical site infection (SSI) in children. This paucity of data is particularly prevalent in low-middle income populations (LMICS), who are likely to be affected most by SSI, yet no prospective, multicentric comparative data exist.

What are the new findings?

- Children undergoing gastrointestinal surgery in lowmiddle development countries are significantly more likely to have SSIs after surgery than their counterparts in high income settings. Use of laparoscopy was associated with lower odds of SSI.

\section{What do the new findings imply?}

- SSI is common in LMICS, occurring in up to a quarter of operations. Our data should be used as a foundation to inform clinical trials and to build initiatives to reduce this. SSI is associated with poorer outcomes and longer lengths of stay, which can be devastating for a family and for children's well-being.

from school and lost working days by parents to assist children, putting family units at risk of catastrophic healthcare expenditure. ${ }^{12}$ Furthermore, the societal burdens of SSI in terms of healthcare costs and the requirements for antibiotic therapy have important consequences for affordability, antibiotic resistance and health system capacity.

Previous studies have predominately come from single hospitals, been heterogeneous in patient and procedure inclusion, and been inconsistent in the different diagnostic criteria for SSI. ${ }^{3-5}$ Furthermore, these studies are often limited in geographical scope, with one systematic review and meta-analysis of HAI identifying only three studies in paediatric patients in low-middle income countries. ${ }^{6}$ In these settings, SSI incidence is markedly higher than in high income countries. ${ }^{7}$ 
The primary aim of this study was to determine the worldwide SSI rate following gastrointestinal surgery in children while the secondary aim was to identify associations between SSI and morbidity among children by country development, according to the United Nation's Human Development Index (HDI).

\section{METHODS}

\section{Patient public involvement}

Patients and the public were involved in design and dissemination of this study, including representation from patients in low HDI countries. We included patients in the conception, design and steering of the overall GlobalSurg 2 Study and GlobalSurg collaborative.

\section{Study design}

This international, multicentre, prospective cohort study was performed according to a published protocol and was registered on ClinicalTrials.gov (NCT02662231). ${ }^{8}$ Investigators were recruited via the international GlobalSurg collaborative research network, surgical associations, training colleges, social media and personal contacts. The structure of surgical collaborative research methodology has been described in detail previously. ${ }^{9}$ Briefly, small teams of local investigators collected data on prospectively determined items, coordinated by regional and national lead investigators, across short time windows, with pooled analysis by a central steering committee. A parallel analysis which also included adults ( $>16$ years) has already been published elsewhere. ${ }^{10}$

This study is reported according to the STrengthening the Reporting of OBservational studies in Epidemiology guidelines and the Statistical Analyses and Methods in the Published Literature. ${ }^{11} 12$

Any hospital in the world performing gastrointestinal surgery on children was eligible to participate in this study. Hospitals could be secondary or tertiary healthcare facilities. Our network includes both small community hospitals and large tertiary referral hospitals. There was no minimum case-volume, or centre-specific requirements to take part. Participating clinicians registered online and had to successfully pass a mandatory online training module to standardise data collection. Investigators included consecutive patients over a 2-week period selected during the course of the study. Multiple non-overlapping 2-week study periods were encouraged. Children $(<16$ years) undergoing elective or emergency gastrointestinal resection were eligible for inclusion. Gastrointestinal resection was defined as complete transection and removal of a segment of the gastrointestinal tract at any point from oesophagus to rectum, including appendix and gall bladder as well as reversal or formation of a stoma.

Data were collected on patient-level risk factors, which included preoperative variables (age, gender, admission to procedure time, American Society of Anesthesiologists (ASA) grade, urgency of operation, presence of malaria or HIV), disease variables (pathology) and operative variables (urgency, procedure start time, operative approach, WHO surgical safety checklist use, antibiotic prophylaxis and intraoperative contamination). Intraoperative contamination was measured by the operating surgeon and defined as follows; clean-contaminated (an incision through the respiratory, alimentary or genitourinary tract under controlled conditions with no direct contamination encountered), contaminated (an operation where there is major break in sterile technique or gross spillage from the gastrointestinal tract, or an incision where acute non-purulent inflammation is encountered or where procedures involve traumatic wounds that have been open for between 12 hours and 24 hours) and dirty (an incision undertaken where viscera are perforated, where acute inflammation or necrosis is encountered, or where there is delayed operation on traumatic wounds) procedures.

Within each team, quality assurance was guaranteed by at least one consultant or attending-level surgeon. Data were recorded prospectively and stored on a secure, internet-based, user-encrypted platform (REDCap). ${ }^{13}$

\section{Primary outcome}

The primary outcome measure was the 30-day postoperative SSI rate according to the Centre for Disease Control and Prevention criteria for SSI. ${ }^{14}$ SSI rates were measured at 30 days following surgery either in person or by computer record/chart review. When 30-day follow-up was not possible, SSI was measured at the point of discharge. Surgical teams were encouraged to assess patients at 30 days (either in-person or via telephone). Secondary outcome measures included the 30-day postoperative mortality rate, reintervention rate, rate of wound organ-space inefction, rate of other HAIs and length of stay. Data were also collected on antimicrobial therapy and microbiology culture from patients with SSI. Antibiotic resistance was defined as resistance in the species which was cultured to the antibiotic administered for prophylaxis.

\section{Statistical analysis}

As described in the protocol, countries were stratified into tertiles of development using the United Nations HDI. This metric is calculated from a composite of country-level statistics including life expectancy, income and education (http://hdr.undp.org/en/statistics). Variation across HDI tertiles were described first using simple summary statistics, including the number of patients and proportions. Continuous variables with normal distributions were summarised using the mean and SD, otherwise the median and IQR was used. Differences were tested using Pearson's $\chi^{2}$ test for categorical variables or the Kruskall-Wallis test for continuous variables. Multilevel logistic regression models were used to adjust for patientlevel (fixed effects) and centre-level (random effects) variations to identify factors which were independently associated with the study outcomes. Effects estimates 


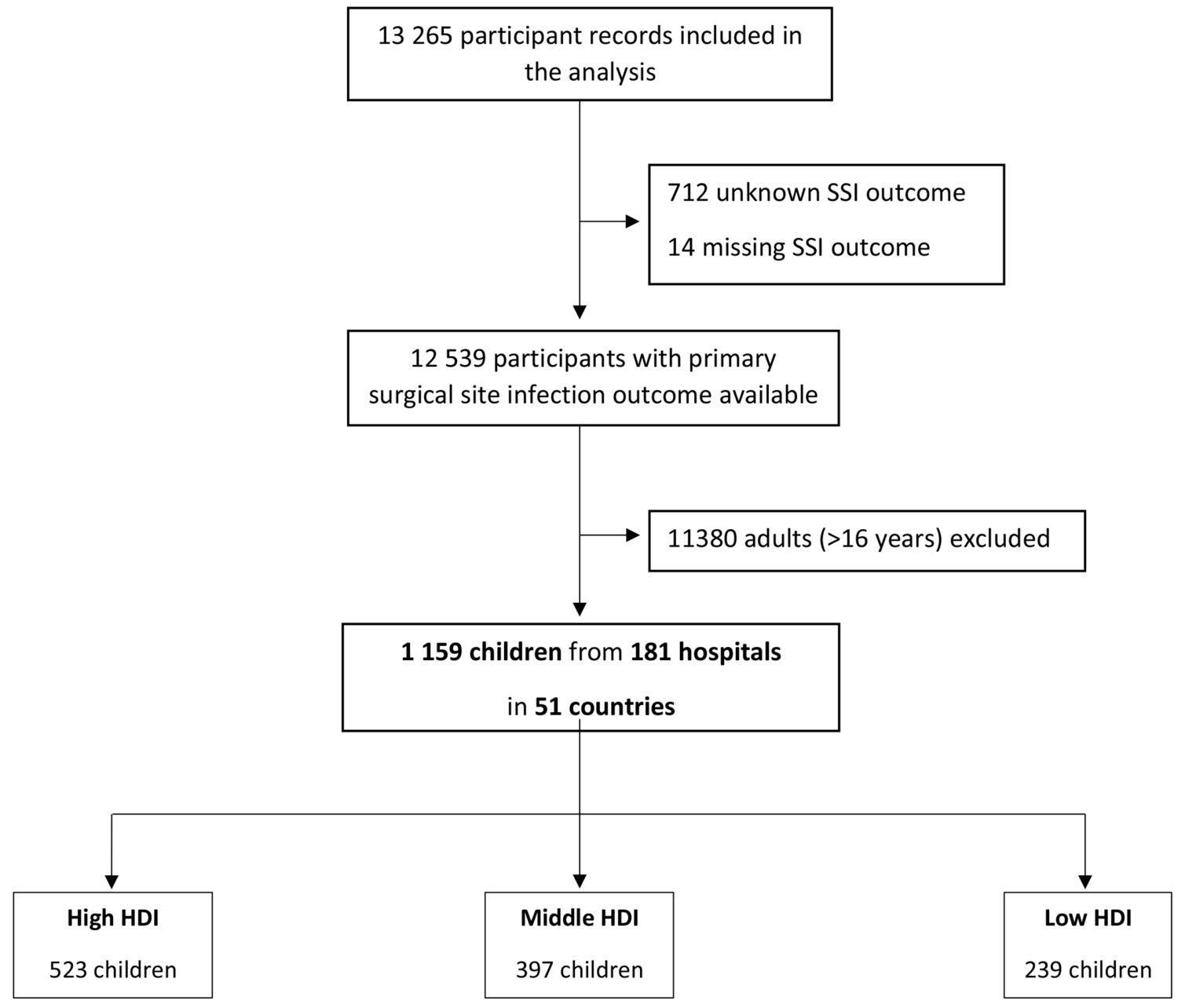

Figure 1 Participant inclusion flow chart. HDI, Human Development Index; SSI, surgical site infection.

from these models are presented as ORs alongside the corresponding 95\% CIs. Clinically plausible and relevant variables were entered into the models, and interactions explored. Model selection was guided through minimisation of the Akaike information criterion (AIC). Statistical significance was taken at the level of $\mathrm{p} \leq 0 \cdot 05$, as specified a priori. All analysis was performed in R V. 3.4.4 (R Foundation for Statistical Computing, Vienna, Austria) using the tidyverse, lme 4 and finalfit packages. As part of this project, an interactive data set explorer is available online at http://ssi.globalsurg.org/.

\section{RESULTS}

Overall 1159 children across 181 hospitals were included from 51 countries during the study period. Of these, 523 $(45 \cdot 1 \%)$ children were from high HDI countries, 397 $(34.2 \%)$ from middle HDI countries and 239 (20.6\%) from low HDI countries. The overall study inclusion flow chart for the GlobalSurg 2 Study and this paediatric subset is shown in figure 1 . There were minimal missing data. Validation of the overall GlobalSurg 2 data set from a sample of 1476 patients found a 93\% correct case ascertainment rate. Data accuracy was high, with Cohen's $\kappa>0.90$ and Pearson's correlation coefficient
0.99 , when data entered at primary entry and validation were compared.

In low HDI settings, patients were younger, with the rate of neonatal surgery fivefold and threefold higher than in high HDI and middle HDI settings, respectively (table 1). Patients in low HDI settings had a preponderance towards being male, with higher ASA grades and were more likely to undergo elective surgery than patients in high HDI settings. Use of laparoscopic surgery was significantly higher in the high HDI group, with surgery more likely to be performed closer to the time of admission. The WHO surgical safety checklist was used in $86.0 \%(450 / 523)$ of patients in high HDI, $43.8 \%(174 / 397)$ of patients in middle HDI and $45.2 \%$ $(81 / 239)$ of patients in low HDI settings. The rate of antibiotic prophylaxis was significantly lower in high HDI settings, despite a higher proportion of dirty procedures performed. Contaminated surgery was most commonly performed in low HDI countries and cleancontaminated procedures in middle HDI countries. Acute appendicitis was the most common indication for surgery across all HDI groups, comprising 70.8\% $(820 / 1159)$ of all cases (online supplemental table S1 and S2). 
BMJ Global Health

Table 1 Patient characteristics by HDI

\begin{tabular}{|c|c|c|c|c|}
\hline & High HDI (n=523) & $\begin{array}{l}\text { Middle HDI } \\
(n=397)\end{array}$ & Low HDI (n=239) & $P$ value \\
\hline \multicolumn{5}{|l|}{ Age } \\
\hline Child (2 years to <12 years) & $274(52.4)$ & $172(43.3)$ & $93(38.9)$ & \\
\hline Infant (1 month to <2 years) & $28(5.4)$ & $46(11.6)$ & $38(15.9)$ & \\
\hline Male & $307(58.7)$ & $212(53.4)$ & $151(63.2)$ & 0.002 \\
\hline Female & $210(40.2)$ & $166(41.8)$ & $84(35.1)$ & \\
\hline Missing & $6(1.1)$ & $19(4.8)$ & $4(1.7)$ & \\
\hline \multicolumn{5}{|l|}{ ASA grade } \\
\hline Missing & $9(1.7)$ & $29(7.3)$ & $4(1.7)$ & \\
\hline \multicolumn{5}{|l|}{ Urgency } \\
\hline Elective & $59(11.3)$ & 79 (19.9) & $65(27.2)$ & $<0.001$ \\
\hline Emergency & $463(88.5)$ & $318(80.1)$ & $174(72.8)$ & \\
\hline Missing & $1(0.2)$ & $0(0.0)$ & $0(0.0)$ & \\
\hline \multicolumn{5}{|l|}{ Operative approach } \\
\hline Open & $235(44.9)$ & $330(83.1)$ & $231(96.7)$ & $<0.001$ \\
\hline Laparoscopic & $288(55.1)$ & $67(16.9)$ & $8(3.3)$ & \\
\hline $6-11$ & $94(18.0)$ & $49(12.3)$ & $33(13.8)$ & \\
\hline $12-23$ & $157(30.0)$ & $60(15.1)$ & $45(18.8)$ & \\
\hline $24-47$ & $76(14.5)$ & $30(7.6)$ & $48(20.1)$ & \\
\hline $48+$ & $37(7.1)$ & $39(9.8)$ & $57(23.8)$ & \\
\hline Missing & $19(3.6)$ & $30(7.6)$ & $13(5.4)$ & \\
\hline \multicolumn{5}{|l|}{ WHO checklist use } \\
\hline No, not available & 43 (8.2) & $146(36.8)$ & $44(18.4)$ & $<0.001$ \\
\hline No, but available & $20(3.8)$ & $74(18.6)$ & $81(33.9)$ & \\
\hline Yes & $450(86.0)$ & $174(43.8)$ & 108 (45.2) & \\
\hline Missing & $10(1.9)$ & $3(0.8)$ & $6(2.5)$ & \\
\hline \multicolumn{5}{|l|}{ Antibiotics: preoperative or prophylactic } \\
\hline No & $80(15.3)$ & $59(14.9)$ & $7(2.9)$ & $<0.001$ \\
\hline Yes & $438(83.7)$ & $333(83.9)$ & $230(96.2)$ & \\
\hline Missing & $5(1.0)$ & $5(1.3)$ & $2(0.8)$ & \\
\hline \multicolumn{5}{|l|}{ Pathology } \\
\hline Appendicitis & $438(83.7)$ & $281(70.8)$ & $101(42.3)$ & $<0.001$ \\
\hline Congenital & $36(6.9)$ & $43(10.8)$ & $79(33.1)$ & \\
\hline
\end{tabular}




\begin{tabular}{|c|c|c|c|c|}
\hline & High HDI $(n=523)$ & $\begin{array}{l}\text { Middle HDI } \\
(n=397)\end{array}$ & Low HDI (n=239) & $P$ value \\
\hline Colitis & $5(1.0)$ & $1(0.3)$ & $2(0.8)$ & \\
\hline Other & $44(8.4)$ & $72(18.1)$ & $57(23.8)$ & \\
\hline \multicolumn{5}{|c|}{ Intraoperative contamination } \\
\hline Clean-contaminated & 347 (66.3) & $314(79.1)$ & $157(65.7)$ & $<0.001$ \\
\hline Contaminated & $63(12.0)$ & 45 (11.3) & $51(21.3)$ & \\
\hline Dirty & 109 (20.8) & $36(9.1)$ & 31 (13.0) & \\
\hline Missing & $4(0.8)$ & $2(0.5)$ & $0(0.0)$ & \\
\hline
\end{tabular}

Data are presented as $n(\%)$. Statistical tests are $\chi^{2}$.

ASA, American Society of Anesthesiologists; HDI, Human Development Index.

The 30-day SSI rate overall was 12.3\% (143/1159). Rates varied by HDI, with an infection rate of $6 \cdot 3 \%(33 / 523)$, $12.8 \%(51 / 397)$ and $24.7 \%(59 / 239)$ in high HDI, middle HDI and low HDI countries, respectively (tables 2 and 3 ). In high HDI settings, SSI was diagnosed at similar rates in-hospital and after discharge (before discharge 2.9\% $(15 / 523)$, after discharge $3.4 \%(18 / 523))$. In middle HDI and low HDI countries, SSI was diagnosed in-hospital more frequently (middle HDI before discharge 6.8\% (27/397), middle HDI after discharge 6.0\% (24/397), low HDI before discharge $18.4 \%$ (44/239), low HDI after discharge $6.3 \%(15 / 239))$.

SSI was significantly more common in contaminated surgery compared with clean-contaminated surgery across all HDI settings $(11.6 \%$ vs $3.5 \%$ in high, $25.9 \%$ vs $9.2 \%$ in middle and $39.0 \%$ vs $17.2 \%$ in low), (online supplemental table S3). Similarly, rates of organ-space infection and HAIs were higher in low HDI settings (table 4). The 30-day mortality and reintervention rates were also significantly higher in patients from low HDI countries when compared with those in middle HDI and high HDI groups. In univariable analyses, low HDI was associated with an increased odds of SSI (onine supplemental table S4). Patient-level factors which were also associated with SSI included non-use of the WHO checklist, the degree of intraoperative contamination and the use of open surgery. When adjusted for clinically plausible explanatory variables, low HDI, intraoperative contamination and open surgery remained significantly associated with higher odds of SSI (figure 2, online supplemental table S4). When HDI was treated as continuous, SSI was correlated with lower country development (figure 3, rank 1 being most developed).

\section{Organisms and antibiotic resistance}

In the $12.3 \%(143 / 1159)$ of patients who developed an SSI, $94.4 \%$ (135/143) had access to microbiological testing and of these $36 \cdot 3 \%(49 / 135)$ had organisms cultured from their wound. The most common organisms were coliforms, present in $46.9 \%$ of culture-positive patients $(23 / 49$, online supplemental table $S 5)$ followed by Staphylococcus aureus species present in $22.4 \%$ of

Table 2 SSI by age and HDI

\begin{tabular}{|c|c|c|c|}
\hline HDI tertile & Age group & No SSI $(n=1016)$ & SSI present $(n=143)$ \\
\hline \multirow[t]{4}{*}{ High } & Adolescent ( 12 years to $<16$ years & $199(96.1)$ & $8(3.9)$ \\
\hline & Child (2 years to <12 years) & $254(92.7)$ & $20(7.3)$ \\
\hline & Infant (1 month to <2 years) & $26(92.9)$ & $2(7.1)$ \\
\hline & Neonate (<1 month) & $11(78.6)$ & $3(21.4)$ \\
\hline \multirow[t]{4}{*}{ Middle } & Adolescent (12 years to <16 years) & $139(88.5)$ & $18(11.5)$ \\
\hline & Child (2 years to <12 years) & $145(84.3)$ & $27(15.7)$ \\
\hline & Infant (1 month to <2 years) & $42(91.3)$ & $4(8.7)$ \\
\hline & Neonate (<1 month) & $20(90.9)$ & $2(9.1)$ \\
\hline \multirow[t]{4}{*}{ Low } & Adolescent ( 12 years to $<16$ years) & $64(88.9)$ & $8(11.1)$ \\
\hline & Child (2 years to <12 years) & $64(68.8)$ & $29(31.2)$ \\
\hline & Infant (1 month to <2 years) & $27(71.1)$ & $11(28.9)$ \\
\hline & Neonate (<1 month) & $25(69.4)$ & $11(30.6)$ \\
\hline
\end{tabular}

Data are presented as $\mathrm{n}(\%)$

HDI, Human Development Index; SSI, Surgical Site Infection. 


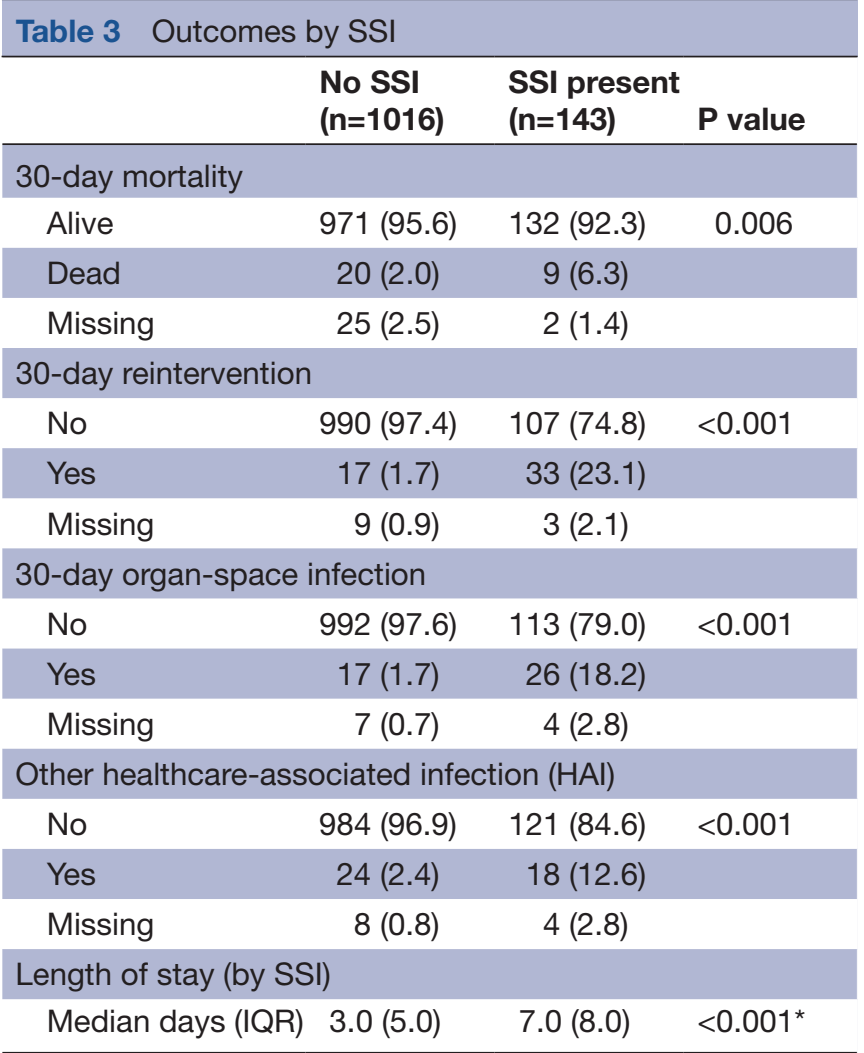

Data are presented as $\mathrm{n}(\%)$. Statistical tests are $\chi^{2}$, besides where the Kruskall-Wallis test was used as denoted by *.

SSI, surgical site infection.

culture-positive patients (11/49, online supplemental table S4). Of those with sensitivity testing performed $(35 / 49), 28.6 \%(10 / 35)$ patients were found to have organisms resistant to antibiotic prophylaxis.

\section{DISCUSSION}

This patient-level, prospective, validated global study found that children in low HDI and middle HDI countries were significantly more likely to suffer from SSI than their counterparts in high HDI countries. In children who did suffer an SSI, there was an association with further complications, including reintervention, and a longer length of stay. Furthermore, we found that children in low-middle income countries had a far higher in-hospital rate of SSI, when compared with children in middle-development and high-development settings.

The main finding of higher incidence and poorer outcomes associated with SSIs in children in low HDI settings likely reflects a complex interplay of potential factors, including weaker preventative measures such as sanitation and vaccination, nutritional issues, shortcomings in knowledge and application of basic infection control, understaffing and overcrowding in medical facilities, absence of local and national guidelines and policies, and the wider lack of structural and financial enablers to improve access to high-quality, affordable healthcare. ${ }^{1516}$ These inequalities are likely to contribute towards healthcare environments which predispose

\begin{tabular}{|c|c|c|c|c|}
\hline & $\begin{array}{l}\text { High HDI } \\
(n=523)\end{array}$ & $\begin{array}{l}\text { Middle HDI } \\
(\mathrm{n}=397)\end{array}$ & $\begin{array}{l}\text { Low HDI } \\
(\mathrm{n}=239)\end{array}$ & $P$ value \\
\hline \multicolumn{5}{|l|}{ SSI } \\
\hline No & $490(93.7)$ & 346 (87.2) & $180(75.3)$ & $<0.001$ \\
\hline Yes & $33(6.3)$ & $51(12.8)$ & $59(24.7)$ & \\
\hline \multicolumn{5}{|c|}{ 30-day mortality } \\
\hline Alive & $504(96.4)$ & $379(95.5)$ & $220(92.1)$ & $<0.001$ \\
\hline Dead & $1(0.2)$ & $13(3.3)$ & $15(6.3)$ & \\
\hline Missing & $18(3.4)$ & $5(1.3)$ & $4(1.7)$ & \\
\hline \multicolumn{5}{|c|}{ 30-day reintervention } \\
\hline No & 503 (96.2) & $379(95.5)$ & $215(90.0)$ & 0.001 \\
\hline Yes & $18(3.4)$ & $11(2.8)$ & $21(8.8)$ & \\
\hline Missing & $2(0.4)$ & $7(1.8)$ & $3(1.3)$ & \\
\hline \multicolumn{5}{|c|}{ 30-day organ-space inefction } \\
\hline No & $502(96.0)$ & $382(96.2)$ & $221(92.5)$ & 0.027 \\
\hline Yes & $16(3.1)$ & $10(2.5)$ & $17(7.1)$ & \\
\hline Missing & $5(1.0)$ & $5(1.3)$ & $1(0.4)$ & \\
\hline \multicolumn{5}{|c|}{ Healthcare-associated infection (HAl) } \\
\hline No & $508(97.1)$ & $381(96.0)$ & $216(90.4)$ & $<0.001$ \\
\hline Yes & $12(2.3)$ & $10(2.5)$ & $20(8.4)$ & \\
\hline Missing & $3(0.6)$ & $6(1.5)$ & $3(1.3)$ & \\
\hline \multicolumn{5}{|c|}{ Length of stay (by HDI) } \\
\hline $\begin{array}{l}\text { Median } \\
\text { days } \\
\text { (IQR) }\end{array}$ & $3.0(4.0)$ & $3.0(5.0)$ & $6.0(6.0)$ & $<0.001^{*}$ \\
\hline
\end{tabular}

Data are presented as $\mathrm{n}(\%)$. Statistical tests are $\chi^{2}$, besides where the Kruskall-Wallis test was used as denoted by *.

HDI, Human Development Index; SSI, surgical site infection.

patients to HAIs, including SSI, which are known contributors to mortality. The contributory inequalities at the point of care have already been well documented in lowmiddle income countries, including a lack of timely access leading to delays in presentation and more advanced disease, and a lack of capacity in surgery and perioperative care compounding the risks of delayed presentation. Lack of postoperative care, including appropriate intensive care facilities, may also contribute to poorer outcomes. Shortages in the staff and facilities to perform routine microbiological testing may result in ineffectual prophylactic antibiotic usage. ${ }^{17}$ We found use of laparoscopy was associated with lower SSI rates, an effect which persisted when HDI was accounted for. Barriers to uptake of surgical technologies (including training, treatment costs and lack of supportive infrastructure for technologies including servicing, equipment support staff, distribution and repair capability), such as laparoscopy, are likely to greatly affect LMICs and increase the observed rate of SSI in children.

The prospective, multicentric design of this research is a major strength, drawing from a large and diverse international patient population. Many global health studies 

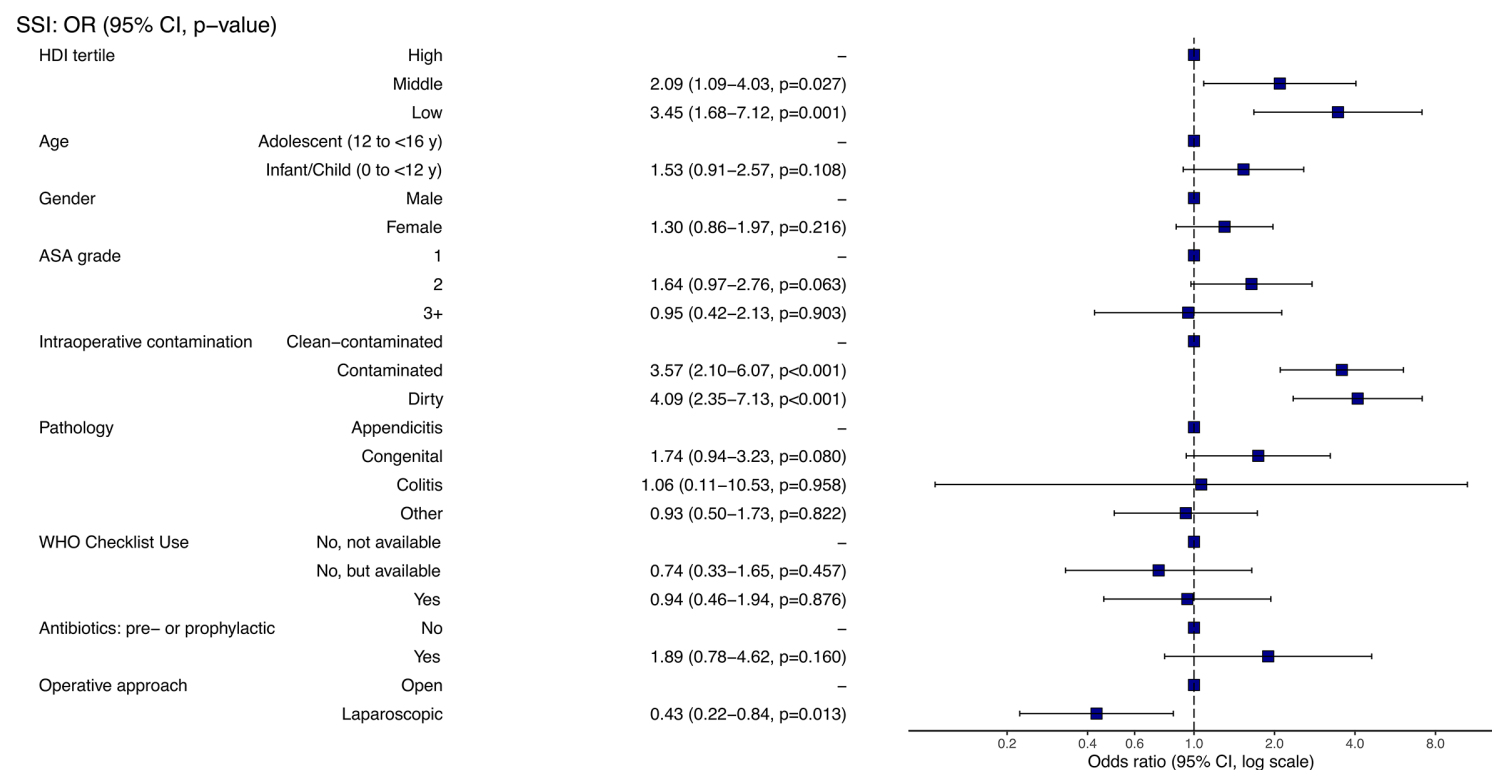

Figure 2 Multilevel, multivariable models for 30-day surgical site infection (SSI) in patients undergoing non-appendicitis surgery (A) or surgery for appendicitis (B). ASA, American Society of Anesthesiologists.

which compare international practice rely on estimates derived from historical data or statistical modelling. Our patient-level approach gives us the ability to look more closely at where SSI is occurring, in which patients, and to identify individuals at the highest risk with far greater power than modelling studies. To explore the effect of HDI on SSI, we constructed risk-adjusted multilevel models which adjusted for patient-level and centre-level effects. In contrast, previous patient-level studies have suffered from low numbers of patients and use heterogeneous diagnostic criteria for SSI. This may explain why we found higher rates of SSI than previous studies. Nonetheless, there are several limitations inherent to our study design, including the inability to follow-up every patient 30 days after surgery. Taking a pragmatic approach given the dispersed global nature of this study, our methodology did not standardise microbiological specimen collection,

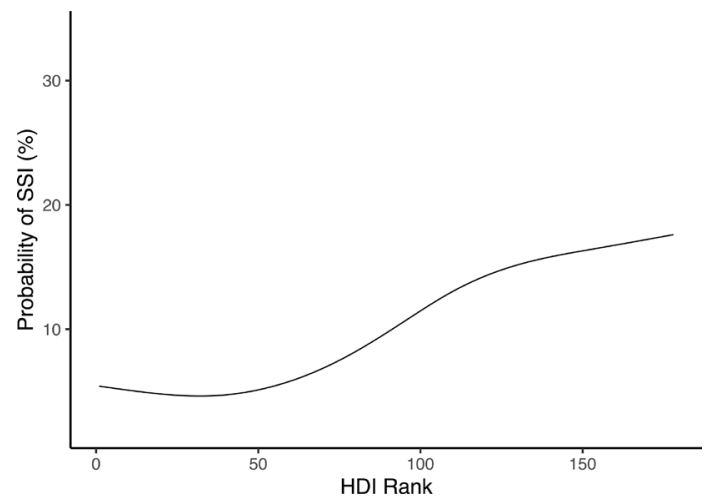

Figure 3 Relationship between HDI rank and probability of SSI (with rank 1 being most highly developed). adjusted for patient-level risk factors including age, sex, use of who checklist, antibiotic prophylaxis, intraoperative contamination and use of laparoscopy. HDI, Human Development Index; SSI, surgical site infection. laboratory assessment, techniques or definitions. We also did not collect centre-level data on the microbiological testing facilities available at each centre. This instead relied on local protocols, including for determining antimicrobial resistance, potentially introducing variation. Although we undertook data validation, there is still the potential for missed cases or inaccurate data. However, the large number of patients, a prospective protocol combined with compulsory investigator training, and the use of local coordinators will have helped minimise any potential bias. A further limitation may be that surgery in children is a highly centralised specialty. In our study, we may have not been able to fully capture the casemix of surgeons in rural settings owing to the requirement for internet to contribute to this study. In high-income countries, smaller centres tend to perform only appendicectomy on children and more complicated surgical cases are referred to a tertiary unit, which may limit the generalisability of our findings.

There is good evidence to support surgical technologies that reduce SSI in abdominal surgery. We identified a significantly lower use of laparoscopic surgery in lowincome and middle-income countries. While this might point to a lack of training, or familiarity with minimal access procedures combined with higher treatment costs, laparoscopic surgery remains one such technology known to reduce SSI. ${ }^{18}$ The most common procedure across all settings was appendicectomy, which can be readily performed using the laparoscopic approach. Our data support that the laparoscopic approach is associated with significantly lower rates of SSI, independent of HDI setting. Given the higher rates of SSI in low-income settings, the absolute benefit of laparoscopy is likely to be even greater than the effect observed in high-income countries. Our previous analysis of both children and adults undergoing surgery suggested a number needed 
to treat, with laparoscopic rather than open surgery, of 6 to prevent one SSI in low HDI countries managing perforated appendicitis. ${ }^{19}$ Therefore, if a low-cost and durable system could be developed to enable greater adoption of laparoscopic surgery for appendicectomy in low HDI settings, this could provide a straightforward means of rapidly reducing SSI through deployment of surgical technologies. ${ }^{20}$

However, propagation of technologies to lowerresource settings will not fully address the underlying, disease-specific and casemix-related reasons for higher SSI rates. In this study, there was a significantly higher proportion of infants and neonates in the low HDI and middle HDI countries, with a significantly higher proportion of neonates undergoing abdominal surgery in low HDI countries. This may reflect the unequal burden of disease in these settings, with a much larger volume of congenital malformations, trauma and other injuries, and infectious causes in low HDI settings, contrasting with high HDI settings, where a wider range of procedures are performed. These are complex procedures, where contamination is frequently encountered, which is reflected in our data. These problems are unlikely to go away soon, as there is a large unmet need and burden of paediatric disease that requires surgery. An estimated $94 \%$ of congenital anomalies occur in low HDI countries, where, due to sociocultural, economic and structural factors, access to high-quality paediatric surgery may be limited. ${ }^{21} 22$ The global burden of childhood trauma and injury, including road traffic collisions, falls, burns, drowning and poisoning, disproportionately affects low-middle income countries (LMICs), and progress addressing this has been slow. ${ }^{23}$ The distribution and incidence of numerous surgically relevant infectious diseases also disproportionately affects LMICs. ${ }^{24}$ Therefore ensuring paediatric surgery in these settings is safe and equitable, and must be made a global health priority.

Given that children make up approximately $50 \%$ of the population in LMICs, ensuring access to safe surgery with good outcomes is vital. However, data describing outcomes following paediatric surgery are lacking in many countries, representing a major research gap and an obstacle to delivering safe surgery with good clinical outcomes. Our data show that paediatric SSI rate in low development and middle development countries is disproportionately higher than in high development settings. As a result, the expected expansion of surgical services in LMICs could have unintended consequences if this disparity in outcomes is not simultaneously addressed. ${ }^{25}$ At the population level, preventing SSI after surgery is also an important component in the global fight against antimicrobial resistance. Several of the WHO recommendations for SSI prevention rely on non-antibiotic mechanisms (eg, preoperative bathing and surgical site sterilisation). Ensuring effective implementation of these may provide a useful way of reducing reliance on antibiotics and consequences such as antimicrobial resistance, however, research on interventions to curb the occurrence of SSI in lower-resource settings is currently extremely limited. ${ }^{26}{ }^{27}$ Future research should aim to quantify the affects of SSI on long-term outcomes in children, particularly the consequences for children in LMICs. Understanding the impact of SSI in these settings on families, educational attainment and developmental outcomes is important to assessing the true burden that these infections have. Furthermore, clinical trials should be undertaken to identify implementable methods of reducing SSI in children across the world, which capture these longer-term outcomes.

\section{CONCLUSIONS}

Worldwide, SSI represents a significant burden of postoperative morbidity in children who receive surgery. Focused initiatives and research, aiming to reduce SSIs in children should be a key priority for global surgery agendas.

Acknowledgements The authors thank the following organisations for assisting in dissemination or translation, or both (alphabetical): Asian Medical Students' Association, Association of Surgeons in Training, College of Surgeons of East, Central \& Southern Africa, Cutting Edge Manipal, Egyptian Medical Student Research Association, International Collaboration For Essential Surgery, International Federation of Medical Student Associations, Italian Society of Colorectal Surgery, Lifebox Foundation, School of Surgery, Student Audit and Research in Surgery, The Electives Network, United Kingdom National Research Collaborative, World Society of Emergency Surgery, World Surgical Association.

Collaborators GlobalSurg Collaborative:Writing Group: Thomas M Drake, Francesco Pata, Dhruv Ghosh, Adesoji 0 Ademuyiwa, Alexis Arnaud, Aneel Bhangu, J Edward Fitzgerald, James Glasbey, Ewen M Harrison Statistical analysia: Thomas M Drake, Ewen M Harrison Steering group: Aneel Bhangu, Adesoji 0 Ademuyiwa, Maria Lorena Aguilera, Philip Alexander, Sara W Al-Saqqa, Giuliano Borda-Luque, Ainhoa Costas-Chavarri, Thomas M Drake, Faustin Ntirenganya, J Edward Fitzgerald, Stuart J Fergusson, James Glasbey, JC Allen Ingabire, Lawani Ismaï, Hosni Khairy Salem, Anyomih Theophilus Teddy Kojo, Marie Carmela Lapitan, Richard Lilford, Andre L Mihaljevic, Dion Morton, Alphonse Zeta Mutabazi, Dmitri Nepogodiev, Adewale 0. Adisa, Riinu Ots, Francesco Pata, Thomas Pinkney, Tomas Poškus, Ahmad Uzair Qureshi, Antonio Ramos-De la Medina, Sarah Rayne, Catherine A Shaw, Sebastian Shu, Richard Spence, Neil Smart, Stephen Tabiri, Ewen M Harrison Study guarantors: Ewen M Harrison (ewen.harrison@ed.ac.uk) Patient representatives: Azmina Verjee, Emmy Runigamugabo Protocol development: Chetan Khatri, Midhun Mohan, Thomas M Drake, James Glasbey, Dmitri Nepogodiev, Catherine A Shaw, Zahra Jaffry, Stuart J Fergusson, Francesco Pata, Adesoji 0 Ademuyiwa, Afnan Altamini, Hosni Khairy Salem, Andrew Kirby, Kjetil Soreide, Gustavo Recinos, Richard Spence, Sarah Rayne, Stephen Tabiri, Jen Cornick, Thomas Pinkney, Richard Lilford, J Edward Fitzgerald, Ewen M Harrison, Aneel Bhangu National leads:Argentina, Maria Marta Modolo; Australia, Dushyant Iyer, Sebastian King, Tom Arthur; Bangladesh, Sayeda Nazmum Nahar; Barbados, Ade Waterman; Benin, Ismail Lawini; Canada, Arnav Agarwal, Augusto Zani, Mohammed Firdouse, Tyler Rouse; China, Qinyang Liu; Colombia, Juan Camilo Correa; Egypt, Hosni Khairy Salem; Estonia, Peep Talving; Ethiopia, Mengistu Worku; France, Alexis Arnaud; Ghana, Stephen Tabiri; Greece, Vassilis Kalles; Guatemala, Maria Lorena Aguilera, Gustavo Recinos; India, Basant Kumar, Sunil Kumar; Indonesia, Radhian Amandito; Ireland, Roy Quek; Italy, Francesco Pata, Luca Ansaloni; Jordan, Ahmed Altibi; Lithuania, Donatas Venskutonis, Justas Zilinskas, Tomas Poskus; Madagascar, John Whitaker; Malawi, Vanessa Msosa; Malaysia, Yong Yong Tew; Malta, Alexia Farrugia, Elaine Borg; Mexico, Antonio Ramos-De la Medina; Morocco, Zineb Bent; Nigeria, Adesoji Ademuyiwa; Pakistan, Tanzeela Gala; Palestinian Territory, Ibrahim Al-Slaibi, Haya Tahboub, Osaid H. Alser; Peru, Diego Romani, Sebestian Shu; Poland, Piotr Major; Romania, Aurel Mironescu, Matei Bratu, Amar Kourdouli; Saint Kitts and Nevis, Aliyu Ndajiwo; Saudi Arabia, Abdulaziz Altwijri, Mohammed Ubaid Alsaggaf, Ahmad Gudal, Al Faifi Jubran; Sierra Leone, Sam Seisay; Singapore, Bettina Lieske; South Africa, Sarah Rayne, Richard Spence; Spain, Irene Ortega; Sri Lanka, Jenifa Jeyakumar, Kithsiri Senanayake; Sudan, 
Omar Abdulbagi; Sweden, Yucel Cengiz; Switzerland, Dmitri Raptis; Turkey, Yuksel Altinel; United Kingdom, Chia Kong, Ella Teasdale, Gareth Irwin, Michael Stoddart, Rakan Kabariti, Sukrit Suresh; United States, Katherine Gash, Mark Scott, Ragavan Narayanan; Zambia, Mayaba Maimbo Local collaborators:Albania: Besmir Grizhja, Shpetim Ymeri, Gezim Galiqi (Spitali Rajonal Shkoder);Argentina: Roberto Klappenbach, Diego Antezana, Alvaro Enrique Mendoza Beleño, Cecilia Costa, Belen Sanchez, Susan Aviles (Hospital Zonal General De Agudos Simplemente Evita); Maria Marta Modolo, Claudio Gabriel Fermani, Ruben Balmaceda, Santiago Villalobos, Juan Manuel Carmona (Hospital Luis C. Lagomaggiore,

Mendoza);Australia: Daniel Hamill, Peter Deutschmann, Simone Sandler, Daniel Cox (Alice Springs Hospital); Ram Nataraja, Claire Sharpin, Damir Ljuhar (Monash Medical Center); Demi Gray, Morgan Haines (Port Macquarie Base Hospital); Dush Iyer, Nithya Niranjan, Scott D'Amours (Liverpool Hospital); Morvarid Ashtari, Helena Franco, (Gold Coast University Hospital).Bangladesh: Ashrarur Rahman Mitul, Sabbir Karim, (Dhaka Shishu (Children) Hospital); Nowrin F. Aman, Mahnuma Mahfuz Estee (Holy Family Red Crescent Medical College \& Hospital); Umme Salma, Joyeta Razzaque, Tasnia Hamidkanta (Dhaka Medical College And Hospital); Sayeedaaktar Tori, Md.Shadid Alamin, Swapnil Roy, Md.Shadid Al Amin (Armed Forces Medical College); Muhtarima Haque, Amreen Faruq, Farhana Iftekhar (Birdem Bangladesh Institute Of Research And Rehabilitation In Diabetes Endocrine And Metabolic Disorder General Hospital);Barbados: Margaret 0'Shea, Greg Padmore, Ramesh Jonnalagadda (Queen Elizabeth Hospital).Belarus: Andrey Litvin, Aliaksandr Filatau, Dzmitry Paulouski, Maryna Shubianok, Tatsiana Shachykava (Gomel Regional Clinical Hospital); Dzianis Khokha, Vladimir Khokha (City Hospital) Benin: Fernande Djivoh, Ismaill Lawani, Francis Dossou (Centre National Hospitalier Et Universitaire Hubert Koutoukou Maga); Djifid Morel Seto, Dansou Gaspard Gbessi, Bruno Noukpozounkou, Yacoubou Imorou Souaibou, (Centre National Hospitalier Et Universitaire Hubert Koutoukou Maga); Kpèmahouton René Keke, Fred Hodonou (Clinique Vignon); Ernestyemalinstephane Ahounou, Thierry Alihonou (Hopital El Fateh); Max Dénakpo, Germain Ahlonsou (Hospital Saint Luc).Botswana: Alemayehu Ginbo Bedada (Princess Marina Hospital).Burundi: Carlos Nsengiyumva, Sandrine Kwizera, Venerand Barendegere (Hopital Militaire De Kamenge).Cambodia: Philip Choi, Simon Stock (World Mate Emergency Hospital)Canada: Luai Jamal, Mohammed Firdouse, Augusto Zani, Georges Azzie, Sameer Kushwaha, Arnav Agarwal (The Hospital For Sick Children).China: Tzu-Ling Chen, Chingwan Yip (Fudan University Affiliated Huashan Hospital).Colombia: Irene Montes, Felipe Zapata, Sebastian Sierra (Clinica CES); Maria Isabel Villegas Lanau, Maria Clara Mendoza Arango, Ivan Mendoza Restrepo (Clinica Las Vegas), Sebastian Sierra, Ruben Santiago Restrepo Giraldo, Mariaclara Mendozaarango (Hospital Universitario San Vicente Fundación).Croatia: Edgar Domini, Robert Karlo, Jakov Mihanovic (Zadar General Hospital).Egypt: Mohamed Youssef, Hossam Elfeki, Waleed Thabet, Aly Sanad, Gehad Tawfik, Ahmed Zaki, Noran Abdel-Hameed, Mohamed Mostafa, Muhammad Fathi Waleed Omar, Ahmed Ghanem, Emad Abdallah, Adel Denewer, Eman Emara, Eman Rashad, Ahmad Sakr, Rehab Elashry, Sameh Emile (Mansoura University Hospital); Toqa Khafagy, Sara Elhamouly, Arwa Elfarargy, Amna Mamdouhmohamed, Ghada Saiednagy, Abeer Esam, Eman Elwy, Aya Hammad, Salwa Khallaf, Eman Ibrahim, Ahmed Saidbadr, Ahmed Moustafa, Amany Eldosoukymohammed, Mohammed Elgheriany, Eman Abdelmageed, Eman Abd Al Raouf, Esraasamir Elbanby, Maha Elmasry, Mahitab Morsyfarahat, Eman Yahyamansor, Eman Magdyhegazy, Esraa Gamal, Heba Gamal, Hend Kandil, Doaa Maherabdelrouf, Mohamed Moaty,(Menofiya University Hospital); Dina Gamal, Nada El-Sagheer, Mohamed Salah, Salma Magdy, Asmaa Salah, Ahmed Essam, Ahmed Ali, Mahmoud Badawy, Sara Ahmed, (Beni Suef University Hospital); Mazed Mohamed, Abdelrahman Assal, Mohamed Sleem, Mai Ebidy, Aly Abdelrazek, Diaaaldin Zahran, Nourhan Adam, Mohamed Nazir, Adel B Hassanein, Ahmed Ismail, Amira Elsawy, Rana Mamdouh, Mohamed Mabrouk, Lopna Ahmed Mohamed Ahmed, Mohamed Hassabalnaby, Eman Magdy, Manar Abd-Elmawla, Marwan Fahim, Bassant Mowafy, Moustafa Ibrahim Mahmoud, Meran Allam, Muhammad Alkelani, Noran Halim El Gendy, Mariam Saad Aboul-Naga, Reham Alaael-Din, Alyaa Halim Elgendy, Mohamed Ismail, Mahmoud Shalaby, Aya Adel Elsharkawy, Mahmoud Elsayed Moghazy, Khaled Hesham Elbisomy, Hend Adel Gawad Shakshouk, Mohamed Fouad Hamed, Mai Mohamed Ebidy, Mostafa Abdelkader, Mohamed Karkeet (Alexandria Main UniversIty Hospital); Hayam Ahmed, Israa Adel, Mohammad Elsayed Omar, Mohamed Ibrahim, Omar Ghoneim, Omar Hesham, Shimaa Gamal, Karim Hilal, Omar Arafa, Sawsan Adel Awad, Menatalla Salem, Fawzia Abdellatif Elsherif, Nourhan Elsabbagh, Moustafa R. Aboelsoud, Ahmed Hossam Eldin Fouad Rida, Amr Hossameldin, Ethar Hany, Yomna Hosny Asar, Nourhan Anwar, Mohamed Gadelkarim, Samar Abdelhady, Eman Mohamed Morshedy, Reham Saad, Nourhan Soliman, Mahmoud Salama (Alexandria Medical Research Institute); Eslam Ezzat, Arwa Mohamed, Arwa Ibrahim, Alaa Fergany, Sara Mohammed, Aya Reda, Yomna Allam, Hanan Adel Saad, Afnan Abdelfatah, Aya Mohamed Fathy, Ahmed El-Sehily, Esraa Abdalmageed Kasem, Ahmed Tarek
Abdelbaset Hassan, Ahmed Rabeih Mohammed, Abdalla Gamal Saad, Yasmin Elfouly, Nesma Elfouly, Arij Ibrahim, Amr Hassaan, Mohammed Mustafa Mohammed, Ghada Elhoseny, Mohamed Magdy, Esraa Abd Elkhalek, Yehia Zakaria, Tarek Ezzat, Ali Abo El Dahab, Mohamed Kelany, Sara Arafa, Osama Mokhtar Mohamed Hassan, Nermin Mohamed Badwi, Ahmad Saber Sleem, Hussien Ahmed, Kholoud Abdelbadeai, Mohamed Abozed Abdullah (Faculty Of Medicine, Zagazig University); Muhammadamsyarauni Lokman, Suraya Bahar, Anan Radyabdelazeam, Abdelrahman Adelshone, Muhammad Bin Hasnan, Athirah Zulkifli, Siti Nur Alia Kamarulzamil, Abdelaziz Elhendawy, Aliang Latif, Ahmad Bin Adnan, Shahadatul Shaharuddin, Aminah Hanum Haji Abdul Majid, Mahmoud Amreia, Dina Al-Marakby, Mahmoud Salma, Mohamadjeffreybin Ismail, Elissa Rifhan Mohd Basir, Citra Dewi Mohd Ali, Aya Yehia Ata (Faculty Of Medicine, Tanta University); Maha Nasr, Asmaa Rezq, Ahmed Sheta, Sherif Tariq, Abdelkhalek Sallam, Abdelrhman KZ Darwish, Sohaila Elmihy, Shady El Hadry, Ahmed Farag, Haidar Hajah, Abdelaziz Seliem, Amro Aglan, Ahmed Zohair, Mahitab Essam, Omar Moussa, Esraa El-Gizawy, Mostafa Samy, Safia Ali, Esraa Elhalawany, Ahmed Ata, Mohamed Elhalawany, Mohamed Nashat, Samar Soliman, Alaa Elazab, Mostada Samy, (El-Menshawy General Hospital); Mohamed A Abdelaziz, Khaled Ibrahim, Ahmed mohamed Ibrahim, Ammar Gado, Usama Hantour (Al-Hussein Hospital); Esraa Alm Eldeen, Mohamed Reda Ioaloa, Arwa Abouzaid, Mostafa Ahmedbahaaeldin, Eman Hashad, Fathy Sroor, Doaa Gamil, Eman Mahmoudabdulhakeem, Mahmoud Zakaria, Fawzy Mohamed, Marwan Abubakr, Elsayed Ali, Hesham Magdy, Mennatallah Ramadan, Mohamed Abdelatymohamed, Salma Mansour, Hager Abdulazizamin, Ahmed Rabiemohamed, Mahmoud Saami, Nada Ahmedredaelsayed, Adham Tarek, Sabry Mohyeldeenmahmoud, Islam Magdy El Sayed, Amira Reda, Martina Yusufshawky, Mohammed Mousasalem, Shahinaz Alaa El-Din, Noha Abdullah Soliman, Muhammed Talaat, Shahinaz Alaael-Dein Ahmed Abdelmoenelhusseiny, Noha Abdullah, Mostafa Ahmedbahaaeldin, Mohammed Elshaar, Aya Abdelfatahibraheem Hager Abdulaziz, Mohammed Kamal Ismail, Mona Hamdymadkor, Mohamed Abdelaty, Sara Mahmoudabdel Kader, Osama Mohamedsalah, (Benha Faculty Of Medicine); Mahmoud Eldafrawy, Ahmed Zakieldeeb, Mostafa Mahmoudeid (October 6 University Hospital); Attia Attia, Khalid Salah El-Dien, Ayman Shwky (Bab El-Shareia University Hospital); Mohamed Adel Badenjki, Abdelrahman Soliman, Samaa Mahmoud Al Attar, (The Memorial Soaad Kafafi University Hospital); Farrag Sayed, Fahd Abdelsabour, Mohammed G. Azizeldine, Muhammad Shawqi, Abdullah Hashim, Ahmed Aamer, Ahmed Mahmoud Abdel Raouf, Mahmoud Abdelshakour, Amal Ibrahim, Basma Mahmoud, Mohamed Ali Mahmoud, Mostafa Qenawy, Ahmed M. Rashed, Ahmed Dahy, Marwa Sayed, Ahmed W. Shamsedine, Bakeer Mohamed, Ahmad Hasan, Mahmoud M. Saad, Khalil Abdulbassit (Assiut University Hospital); Nadia Khalidabdelatif, Nada Elzahed, Ahmed Elkashash, Nada mohamed bekhet, Sarah Hafez, Ahmed Gad, Mahmoud Elkhadragy Maher, Ahmed Abdelsameea, Mohamed Hafez, Ahmad Sabe, Ataa Ahmed, Ahmed Shahine, Khaled Dawood, Shireen Gaafar, Reem Husseiny, Omnia Aboelmagd, Ahmed Soliman, Nourhan Mesbah, Hossam Emadeldin, Amgad Almeligy, Amira Hassan Bekhet, Doaa Hasan, Khaled Alhady, Ahmad Khaled Sabe, Mahmoud A. Elnajjar, Majed Aboelella, Ward Hamsho, Ihab Hassan, Hala Saad, Galaleldin Abdelazim, Hend Mahmoud, Noha Wael, Ahmedali M Kandil, Ahmed Magdy, Shimaa Saidelkholy, Badreldineadel Adel, Kareem Dabbour, Saged Elsherbiney, Omar Mattar, Abdulshafi Khaled Abdrabou, Mohammed Yahia mohamed Aly, Abdelrahman Geuoshy, Ahmedglal Elnagar, Saraibrahim Ahmed, Ibrahem Abdelmotaleb, Amr Ahmed Saleh, Hesham Mohammedbakry, Manar Saeed, Shady Mahmoud, Badreldinadel Tawfik, Samar Adel Ismail, Esraay Zakaria, Mariam 0.Gad, Mohamedsalah Elhelbawy, Monica Bassem, Nadia Khaledabdel-Latif, Noha Maraie, Nourhan Medhat Elhadary, Nourhan Semeda, Shaza Rabiemohamed, Hesham Mohammed Bakry, AA Essam (Kasr Al-Ainy Faculty Of Medicine, Cairo University); Dina Tarek, Khlood Ashour, Alaa Elhadad, Abdulrahman Abdel-Aty, Ibrahim Rakha, Sara Mamdouh Matter, Rasha Abdelhamed, Omar Abdelkader, Ayat Hassaan, Yasmin Soliman, Amna Mohamed, Sara Ghanem, Sara Amr Mohamed Farouk, Eman Mohamed Ibrahim, Esraa El-Taher (Faculty Of Medicine Seuz Canal University); Merna Mostafa, Mohamedfawzy Mahrousbadr, Rofida Elsemelawy Aya El-Sawy, Ahmad Bakr, Ahmad Abdel Razaq Al Rafati (Smouha University Hospital); Estonia: Sten Saar, Arvo Reinsoo, Peep Talving (The North Estonia Medical Centre);Ethiopia: Nebyou Seyoum, Tewodros Worku, Agazi Fitsum, (Addis Ababa University, College Of Health Sciences, School of Medicine); Finland: Matti Tolonen, Ari Leppaniemi, Ville Sallinen (Helsinki University Hospital); France: Benoit Parmentier, Matthieu Peycelon, Sabine Irtan (Trousseau Hospital, APHP); Sabrina Dardenne, Elsa Robert (GHICL); Betty Maillot, Etienne Courboin, Alexis Pierre Arnaud, Juliette Hascoet (CHU Rennes); Olivier Abbo, Amir Ait Kaci, Thomas Prudhomme (CHU Toulouse); Quentin Ballouhey, Celine Grosos, Laurent Fourcade (CHU Limoges); Tolg Cecilia, Colombani Jean-Francois, FrancoisCoridon Helene (CHU Martinique); Xavier Delforge, Elodie Haraux (CHU Amiens Picardie); Bertrand Dousset, Roberto Schiavone, Sebastien Gaujoux, (Cochin APHP); Jean-Baptiste Marret, Aurore Haffreingue, Julien Rod (CHU Caen); Mariette 
Renaux-Petel (CHU Rouen); Jean-Francois Lecompte, Jean Breaud, Pauline Gastaldi (CHU Lenval Nice); Chouikh Taieb, Raquillet Claire, Echaieb Anis (Hopital Robert Ballanger, Paris); Nasir Bustangi, Manuel Lopez, Aurelien Scalabre (CHU De Saint Etienne); Maria Giovanna Grella (CHU Poitiers); Aurora Mariani, Guillaume Podevin, Francoise Schmitt (CHU Angers); Erik Hervieux, Aline Broch, Cecile Muller (Hopital Necker Enfants Malades, APHP).Ghana: Stephen Tabiri, Anyomih Theophilus Teddy Kojo, Dickson Bandoh, Francis Abantanga, Martin Kyereh, Hamza Asumah, Eric Kofi Appiah, Paul Wondoh (Tamale Teaching Hospital); Adam Gyedu, Charles Dally, Kwabena Agbedinu Michael Amoah, Abiboye Yifieyeh, Kwabena Agbedinu (Kwame Nkrumah University of Science and Technology/Komfo Anokye Teaching Hospital); Frank Owusu (St. Particks Hospital); Mabel Amoako-Boateng, Makafui Dayie, Richmond Hagan, Sam Debrah, (Cape Coast Teaching Hosital); Micheal OheneYeboah, Joe-Nat Clegg-Lampety, Victor Etwire, Jonathan Dakubo, Samuel Essoun, William Bonney, Hope Glover-Addy, Samuel Osei-Nketiah, Joachim Amoako, Niiarmah Adu-Aryee, William Appeadu-Mensah, Antoinette Bediako-Bowan, Florence Dedey (Korle Bu Teaching Hospital); Mattew Ekow, Emmanuel Akatibo, Musah Yakubu (Baptist Medical Center, Nalerigu); Hope Edem Kofi Kordorwu, Kwasi Asare-Bediako, Enoch Tackie (Keta Hospital District Hospital, Keta); Kenneth Aaniana, Emmanuel Acquah, Richard Opoku-Agyeman, Anthony Avoka, Kwasi Kusi, Kwame Maison, (Techiman Holy Family Hospital); Frank Enoch Gyamfi (Berekum Holy Family Hospital); Gandau Naabarnabas, Saiba Abdul-Latif (Upper West Regional Hospital); Philip Taahamoako (Samapa Government Hospital); Anthony Davor, Victor Dassah (Upper East Regional Hospital); Enoch Dagoe (St. Mary's Hospital); Prince Kwakyeafriyie (Essumejaman Sda Hospital, Dominase); Elliot Akoto, Eric Ackom, Ekow Mensah (Dormaa Presbyterian Hospital); Ebenezer Takyi Atkins, Christian Lari Coompson (Brongho-Ahafo Regional Hospital, Sunyani) Greece: Nikolaos Ivros, Christoforos Ferousis, Vasileios Kalles, Christos Agalianos, Ioannis Kyriazanos, Christos Barkolias, Angelos Tselos, Georgios Tzikos, Evangelos Voulgaris (Naval And Veterans Hospital); Dimitrios Lytras, Athanasia Bamicha, Kyriakos Psarianos (Achillopoyleio General Hospital Of Volos); Anastasios Stefanopoulos (General Hospital Of Nafplio, Department Of Surgery); Ioannis Patoulias, Dimitrios Sfougaris, Ioannis Valioulis (G. Gennimatas Hospital); Dimitrios Balalis, Dimitrios Korkolis, Dimitrios K Manatakis (Saint Savvas Cancer Hospital); Georgios Kyrou, Georgios Karabelias, lason-Antonios Papaskarlatos (General And Oncological Hospital Of Kifissia-Athens); Kolonia Konstantina, Nikolaos Zampitis, Stylianos Germanos (General Hospital Of Larissa); Aspasia Papailia, Theodosios Theodosopoulos, Georgios Gkiokas (2nd Dept Of Surgery, Aretaieion Hospital, National \& Kapodistrian University Of Athens School Of Medicine); Magdalini Mitroudi, Christina Panteli, Thomas Feidantsis, Konstantinos Farmakis, (G. Gennimatas General Hospital); Dimitrios Kyziridis, Orestis loannidis, Styliani Parpoudi (4th Surgical Department, Aristotle University Of Thessaloniki, General Hospital, Papanikolaou); Georgios Gemenetzis, Stavros Parasyris (Attikon University Hospital); Christos Anthoulakis, Nikolaos Nikoloudis, Michail Margaritis (Serres General Hospital);Guatemala: Maria-Lorena Aguilera-Arevalo, Otto Coyoy-Gaitan, Javier Rosales (Hospital General San Juan De Dios); Luis Tale, Rafael Soley, Emmanuel Barrios (Juan Jose Arevalo Bermejo); Servio Tulio Torres Rodriguez, Carlos Pazgalvez, Danilo Herreracruz (Hospital San Vicente); Guillermo Sanchez Rosenberg, Alejandro Matheu, David Monterroso Cohen (Hospital Herrera Llerandi);Haiti: Marie Paul, Angeline Charles (Hopital Universitaire De Mirebalais); Hong Kong SAR, China: Justin Chak Yiu Lam, Man Hon Andrew Yeung, Chi Ying Jacquelyn Fok, Ka Hin Gabriel Li, Anthony Chuk-Him Lai, Yuk Hong Eric Cheung, Hong Yee Wong, Ka Wai Leung, Tien Seng Bryan Lee, Wai Him Lam, Weihei Dao, Stephanie Hiu-wai Kwok, Tsz-Yan Katie Chan, Yung Kok Ng, (Prince Of Wales Hospital); Qinyang Liu, Chi Chung Foo, James Yang, (University Of Hong Kong);India: Basant Kumar, Ankur Bhatnagar, Vijaid Upadhyaya, (Sanjay Gandhi Post Graduate Institute Of Medical Sciences); Sunil Kumar (Excelcare Hospital); Uday Muddebihal, Wasim Dar, Janardhan Kc (Manipal Hospitals); Philip Alexander, Neerav Aruldas, (Lady Willingdon Hospital);Indonesia: Fidelis Jacklyn Adella, Anthonius Santoso Rulie, Ferdy Iskandar, Jonny Setiawan (Atma Jaya Hospital); Cicilia Viany Evajelista, Hani Natalie, Arlindawati (Dr. Oen Surakarta Hospital); Rudy Gunawan, Herlin Karismaningtyas, Lusipadmasulistianingsih Mata, Ferryfitriyaayu Andika, Afifatun Hasanah, T Ariani Widiastini, Nurlailaayu Purwaningsih, Annisa Dewi Fitriana Mukin, Dinafaizatur Rahmah, Hazmidwinanda Nurqistan, Hasbimaulana Arsyad, Novia Adhitama (Rsd Dr Soebandi); Wifantosaditya Jeo, Nathania Sutandi, Audrey Clarissa, Phebe Anggita Gultom, Matthew Billy, Andreass Haloho, Radhian Amandito, Nadya Johanna, Felix Lee (Rsupn Cipto Mangunkusumo);Ireland: Radin Mohd Nurrahman Radin Dorani, Martha Glynn, Mohammad Alherz, Wennweoi Goh, Haaris A. Shiwani, Lorraine Sproule, Kevin C. Conlon (Tallaght Hospital, Trinity College Dublin)Israel: Miklosh Bala, Asaf Kedar (Hadassah Hebrew University Medical Center);Italy: Luca Turati, Federica Bianco, Francesca Steccanella, (Treviglio Hospital); Gaetano Gallo, Mario Trompetto, Giuseppe Clerico (Department of Colorectal Surgery, S. Rita Clinic, Vercelli); Matteo Papandrea, Giuseppe Sammarco,
Rosario Sacco (Department of Medical and Surgical Sciences, Policlinico Universitario Mater Domini Campus Salvatore Venuta, Catanzaro); Angelo Benevento, Francesco Pata, Luisa Giavarini (Sant'Antonio Abate Hospital, Gallarate); Mariano Cesare Giglio, Luigi Bucci, Gianluca Pagano, Viviana Sollazzo, Roberto Peltrini, Gaetano Luglio (Federico II University Of Naples); Arianna Birindelli, Salomone Di Saverio, Gregorio Tugnoli (Maggiore Hospital); Miguel Angel Paludi, Pietro Mingrone, Domenica Pata (Nicola Giannettasio Hospital, Rossano); Francesco Selvaggi, Lucio Selvaggi, Gianluca Pellino, Natale Di Martino (Universitá della Campania "Luigi Vanvitelli", Naples); Gianluca Curletti, Paolo Aonzo, Raffaele Galleano (Ospedale Santa Corona, Pietra Ligure (SV)); Stefano Berti, Elisa Francone, Silvia Boni, (S. Andrea Hospital, Poll-Asl 5, La Spezia), Laura Lorenzon, Annalisa Lo Conte, Genoveffa Balducci (Sant'Andrea Hospital, Sapienza University of Rome); Gianmaria Confalonieri, Giovanni Pesenti (Azienda Ospedaliera Alessandro Manzoni); Laura Gavagna, Giorgio Vasquez, Simone Targa, Savino Occhionorelli, Dario Andreotti (Azienda 0spedaliero-Universitaria Di Ferrara); Giacomo Pata (A.0 Spedali Civili Di Brescia); Fabrizio Aquilino, Nicola Chetta, Arcangelo Picciariello (Azienda Ospedaliero Universitaria Consorziale Policlinico Di Bari); Mohamed Abdelkhalek, Andrea Belli, Silvia De Franciscis (Istituto Nazionale Tumori Fondazione, Pascale-I.R.C.C.S.); Annamaria Bigaran, Alessandro Favero, Stefano M.M Basso (Azienda Per L'assistenza Sanitaria N. 5 Friuli Occidentale); Paola Salusso, Martina Perino, Sylvie Mochet, Diego Sasia, Francesco Riente, Marco Migliore (Azienda Sanitaria 0spedaliera San Luigi Gonzaga); David Merlini, Silvia Basilicò, Carlo Corbellini (Ospedale Di Rho - ASST Rhodense); Veronica Lazzari, Yuri Macchitella, Luigi Bonavina (IRCCS Policlinico San Donato); Daniele Angelieri, Diego Coletta, Federica Falaschi, Marco Catani, Claudia Reali, Mariastella Malavenda, Celeste Del Basso, Sergio Ribaldi, Massimo Coletti, Andrea Natili, Norma Depalma, Immacolata lannone, Angelo Antoniozzi, Davide Rossi (Policlinico Umberto I Emergency Surgery Department); Daniele Gui, Gerardo Perrotta, Matteo Ripa, Francesco Ruben Giardino, Maurizio Foco, (Fondazione Policlinico Universitario Agostino Gemelli); Erika Vicario, Federico Coccolini, Luca Ansaloni, Gabriela Elisa Nita (A0 Papa Giovanni XXIII); Nicoletta Leone, Andrea Bondurri, Anna Maffioli (Ospedale Sacco); Andrea Simioni, Davide De Boni, Sandro Pasquali (IOV - Istituto Oncologico Veneto); Elena Goldin, Elena Vendramin, Eleonora Ciccioli (Azienda Ospedaliera di Padova); Umberto Tedeschi, Luca Bortolasi, Paola Violi, Tommaso Campagnaro, Simone Conci, Giovanni Lazzari, Calogero lacono, Alfredo Guglielmi, Serena Manfreda (Azienda Ospedaliera Universitaria Integrata di Verona); Anna Rinaldi, Maria Novella Ringressi, Beatrice Brunoni (Azienda Ospedaliera Universitaria Careggi); Giuseppe Salamone, Mirko Mangiapane, Paolino De Marco, Antonella La Brocca, Roberta Tutino, Vania Silvestri, Leo Licari, Tommaso Fontana, Nicolò Falco Gianfranco Cocorullo, (Policlinico Paolo Giaccone di Palermo); Mostafa Shalaby, Pierpaolo Sileri, Claudio Arcudi (Policlinico Tor Vergata Hospital, Rome) Jordan: Isam Bsisu, Khaled Aljboor, Lana Abusalem, Aseel Alnusairat, Ahmad Qaissieh, Emad Al-Dakka, Ali Ababneh, Oday Halhouli (Jordan University Hospital);Kenya: Taha Yusufali, Hussein Mohammed (Kenyatta National Hospital); Justus Lando, Robert Parker, Wairimu Ndegwa (Tenwek Hospital);Lithuania: Mantas Jokubauskas, Jolanta Gribauskaite, Donatas Venskutonis (Lithuanian University Of Health Sciences); Justas Kuliavas, Audrius Dulskas, Narimantas E. Samalavicius (Klaipeda University Hospital, National Cancer Institute); Kristijonas Jasaitis, Audrius Parseliunas, Viktorija Nevieraite, Margarita Montrimaite, Evelina Slapelyte, Edvinas Dainius, Romualdas Riauka, Zilvinas Dambrauskas Andrejus Subocius, Linas Venclauskas, Antanas Gulbinas, Saulius Bradulskis, Simona Kasputyte, Deimante Mikuckyte, Mindaugas Kiudelis, Justas Zilinskas, Tomas Jankus, Steponas Petrikenas (Lithuanian University of Health Sciences); Matas Pazusis, Zigmantas Urniezius, Mantas Vilcinskas (Republican Hospital of Kaunas); Vincas Jonas Banaitis, Vytautas Gaizauskas, Edvard Grisin, Povilas Mazrimas, Rokas Rackauskas, Mantas Drungilas, Karolis Lagunavicius, Vytautas Lipnickas, Dovile Majauskyte, Valdemaras Jotautas, Tomas Abaliksta, Laimonas Uscinas, Gintaras Simutis, Adomas Ladukas, Donatas Danys, Erikas Laugzemys, Saulius Mikalauskas, Tomas Poskus, Elena Zdanyte Sruogiene, (Vilnius University Hospital); Petras Visinskas, Reda Zilinskiene, Deividas Dragatas (Hospital Of Jonava); Andrius Burmistrovas, Zygimantas Tverskis (Taurage Hospital); Arturas Vaicius, Ruta Mazelyte, Antanas Zadoroznas (Viesoji Istaiga Rokiskio Rajono Ligonine); Nerijus Kaselis, Greta Ziubrytė, (Republican Hospital Of Klaipeda);Madagascar: Finaritra Casimir Fleur Prudence Rahantasoa, Luc Herve Samison, Fanjandrainy Rasoaherinomenjanahary, Todisoa Emmanuella Christina Tolotra (Joseph Ravoahangy Andrianavalona Hospital);Malawi: Cornelius Mukuzunga, Vanessa Msosa, Chimwemwe Kwatiwani, Nelson Msiska (Kamuzu Central Hospital);Malaysia: Feng Yih Chai, Siti Mohd Desa Asilah, Khuzaimah Zahid Syibrah (Hospital Keningau); Pui Xin Chin, Afizah Salleh, Nur Zulaika Riswan (Kajang Hospital); April Camilla Roslani, Hoong-Yin Chong, Nora Abdul Aziz, Keat-Seong Poh, Chu-Ann Chai, Sandip Kumar (University Malaya Medical Centre); Mustafa Mohammed Taher, Nik Ritza Kosai, Dayang Nita Abdul Aziz, Reynu Rajan (Universiti Kebangsaan Malaysia Medical Centre UKMMC); 
Rokayah Julaihi, Durvesh Lacthman Jethwani, Muhammad Taqiyuddin Yahaya, Nik Azim Nik Abdullah, Susan Wndy Mathew, Kuet Jun Chung, Milaksh Kumar Nirumal, R. Goh Ern Tze, Syed Abdul Wahhab Eusoffee Wan Ali (Sarawak General Hospital); Yiing Yee Gan, Jesse Ron Swire Ting (Hospital Sibu); Samuel S. Y. Sii, Kean Leong Koay, Yi Koon Tan, Alvin Ee Zhiun Cheah, Chui Yee Wong, Tuan Nur'Azmah Tuan Mat, Crystal Yern Nee Chow, Prisca A.L. Har, Yishan Der (Hospital Sultanah Aminah); Yong Yong Tew, Fitjerald Henry, Xinwei Low (Selayang Hospital); Ya Theng Neo, Hian Ee Heng, Shu Ning Kong, Cheewei Gan, Yi Ting Mok, Yee Wen Tan, Kandasami Palayan, Mahadevan Devatata, Yih Jeng Cheong (Hospital Tuanku Jaafar); Kuhaendran Gunaseelan, Wan Nurul Ain Wan Mohd Nasir, Pigeneswaren Yoganathan, (Hospital Keningau); Eu Xian Lee, Jian Er Saw, Li Jing Yeang, Pei Ying Koh, Shyang Yee Lim, Shuang Yi Teo (Hospital Pulau Pinang / Penang Medical College);Malta: Nicole Grech, Daniela Magri, Kristina Cassar, Christine Mizzi, Malcolm Falzon, Nihaal Shaikh, Ruth Scicluna, Stefan Zammit, Elaine Borg, Sean Mizzi, Svetlana Doris Brincat, Thelma Tembo, Vu Thanh Hien Le, Tara Grima, Keith Sammut, Kurt Carabott, Alexia Farrugia, Ciskje Zarb, Andre Navarro, Thea Dimech, Georgettemarie Camilleri, Isaac Bertuello, Jeffrey Dalli, Karl Bonavia (Mater Dei Hospital);Mexico: Samantha Corro-Diaz, Marisol Manriquez-Reyes, Antonio Ramos-De la Medina (Hospital Espanol de Veracruz);Morocco: Amina Abdelhamid, Abdelmalek Hrora, Sarah Benammi, Houda Bachri, Meryem Abbouch, Khaoula Boukhal, Redouane Mammar Bennai, Abdelkader Belkouchi, Mohamed Sobhi Jabal, Chaymae Benyaiche (IBN Sina Hospital)Netherlands: Maarten Vermaas, Lucia Duinhouwer, (ljsselland Hospital)Nicaragua: Javier Pastora, Greta Wood, Maria Soledad Merlo (Hospital Escuela Oscar Danilo Rosales Arguello);Nigeria: Akinlabi Ajao, Omobolaji Ayandipo, Taiwo Lawal, Abdussemiu Abdurrazzaaq, (University College Hospital, Ibadan); Olufemi Habeeb, Muslimat Alada, Abdulrasheed Nasir, James Adeniran, Olufemi Habeeb, Ademola Popoola, Ademola Adeyeye (University Of llorin Teaching Hospital,Ilorin); Ademola Adebanjo, Opeoluwa Adesanya, Adewale Adeniyi (Federal Medical Centre, Abeokuta,); Henry Mendel, Bashir Bello, Umar Muktar (Usmanu Danfodiyo University Teaching Hospital); Adedapo Osinowo, Thomas Olagboyega Olajide, Oyindamola Oshati, George Ihediwa, Babajide Adenekan, Victor Nwinee, Felix Alakaloko, Adesoji Ademuyiwa, Olumide Elebute, Abdulrazzaq Lawal, Chris Bode, Mojolaoluwa Olugbemi (Lagos University Teaching Hospital); Alaba Adesina, Olubukola Faturoti, Oluwatomi Odutola, Oluwaseyi Adebola, Clement Onuoha, Ogechukwu Taiwo (Babcock University Teaching Hospital); Omolara Williams, Fatai Balogun, Olalekan Ajai, Mobolaji Oludara, lloba Njokanma, Roland Osuoji (Lagos State University Teaching Hospital); Stephen Kache, Jonathan Ajah, Jerry Makama (Barau Dikko Teaching Hospital, Kaduna State University, Kaduna); Ahmed Adamu, Suleiman Baba, Mohammad Aliyu, Shamsudeen Aliyu, Yahaya Ukwenya, Halima Aliyu, Tunde Sholadoye, Muhammad Daniyan, Oluseyi Ogunsua (Ahmadu Bello University Teaching Hospital Zaria); Lofty-John Anyanwu, Abdurrahaman Sheshe, Aminu Mohammad (Aminu Kano Teaching Hospital); Samson Dr Olori, Philip Mshelbwala, Babatunde Odeyemi, Garba Samson, Oyediran Kehinde Timothy, Sani Ali Samuel (University Of Abuja Teaching Hospital); Anthony Ajiboye, Ademola Adeyeye, Isaac Amole, Olajide Abiola, Akin Olaolorun (Bowen University Teaching Hospital);Norway: Kjetil Søreide, Torhild Veen, Arezo Kanani, Kristian Styles, Ragnar Herikstad, Johannes Wiik Larsen, Jon Arne Søreide (Stavanger University Hospital); Elisabeth Jensen, Mads Gran, Eirik Kjus Aahlin (University Hospital Of Northern Norway); Tina Gaarder, Peter Wiel Monrad-Hansen, Pål Aksel Næss (Oslo University Hospital); Giedrius Lauzikas, Joachim Wiborg, Silje Holte (Sykehuset Telemark HF); Knutmagne Augestad, Gurpreetsingh Banipal, Gurpreetsingh Banipal, Michela Monteleone, Thomastetens Moe, Johanneskurt Schultz (Akershus University Hospital);Palestine: Taher Al-taher, Ayah Hamdan, Ayman Salman, Rana Saadeh, Aseel Musleh, Dana Jaradat, Soha Abushamleh, Sakhaa Hanoun, Amjad Abu Qumbos, Aseel Hamarshi, Ayman And Taher (Al Makassed Islamic Charitable Society Hospital, Jerusalem); Israa Qawasmi, Khalid Qurie, Marwa Altarayra, Mohammad Ghannam, Alaa Shaheen, Azher Herebat (Alia Governmental Hospital); Aram Abdelhaq, Ahmad Shalabi, Maram Abu-toyour, Fatema Asi, Ala Shamasneh, Anwar Atiyeh, Mousa Mustafa, Rula Zaatreh, Majd Dabboor (Palestine medical Complex); Enas Alaloul, Heba Baraka, Jehad Meqbil, Alaa Al-buhaisi, Mohamedraed Elshami, Samah Afana, Sahar Jaber, Said Alyacoubi, Yousef Abuowda (Islamic University of Gaza Medical School, European Gaza Hospital \& Shifa Hospital); Tasneem Idress, Eman Abuqwaider (Mizan hospital); Sara Al-saqqa, Alaa Bowabsak, Alaa Eljamassi, Doaa Hasanain, Hadeel Al-farram, Maram Salah, Aya Firwana, Marwa Hamdan, Israa Awad (Al-Shifa Hospital); Ahmad Ashour, Fayez Elian Al Barrawi (Bit Hanoun Hospital); Ahmed Al-khatib, Maha Al-faqawi, Mohamed Fares (Nasser Hospital); Amjad Elmashala, Mohammad Adawi, Ihdaa Adawi (Beit Jala Governmental Hospital); Reem Khreishi, Rose Khreishi (Martyr Thabet Govermental hospital, Tulkarem); Ahmad ashour, Ahed ghaben (Indonesian Hospital, Gaza)Pakistan: Najwa Nadeem, Muhammad Saqlain (Allied Hospital, Faisalabad); Jibran Abbasy, Abdul Rehman Alvi, Tanzeela Gala, Noman Shahzad (Aga Khan University); Kamran Faisal Bhopal, Zainab Iftikhar, Muhammad Talha Butt; Syed
Asaat UI Razi, Asdaq Ahmed, Ali Khan Niazi (Bahawal Victoria Hospital); Ibrahim Raza, Fatima Baluch, Ahmed Raza, Ahmad Bani-Sadar, Ahmad Uzair Qureshi, Muhammad Adil, Awais Raza, (King Edward Medical University, Mayo Hospital, Lahore); Mahnoor Javaid, Muhammad Waqar, Maryamali Khan (CMH Lahore Medical And Dental College); Mohammadmohsin Arshad, Mohammadasim Amjad (Nishtar Medical College And Hospital);Paraguay: Gustavo Miguel Machain Vega, Jorge Torres Cardozo, Marcelo OHiggins Roche, Gustavo Rodolfo Pertersen Servin, Helmut Alfredo Segovia Lohse, Larissa Ines Paez lopez, Ramon Augusto Melo Cardozo (Hospital de Clínicas, II Catedra de Clinica Quirurgica, Universidad Nacional de Asuncion)Peru: Fernando Espinoza, Angel David Perez Rojas, Diana Sanchez, Camila Sanchez Samaniego, Shalon Guevara Torres, Alexander Canta Calua, Cesar Razuri, Nadia Ortiz, Xianelle Rodriguez, Nahilia Carrasco, Fridiz Saravia, Hector Shibao Miyasato, Maria Valcarcel-Saldana, Ysabel Esthefany Alejos Bermudez, Juan Carpio, Walter Ruizpanez, Pedroangel Toribioorbegozo, (Hospital Nacional Arzopispo Loayza); Carolina Guzman Duenas, Kevin Turpo Espinoza, Ana Maria Sandoval Barrantes, Jorge Chungui, Sebastian Shu, Lorena Fuentes-Rivera, Carmen Fernandez, Diego Romani, Barbara, Joselyn Ye (Hospital National Cayetano Heredia); Ricardo Velasquez, Jannin Salcedo (Clínica De Especialidades Médicas); Ana Lucia Contreras-Vergara, Angelica Genoveva Vergara Mejia, Maria Soledad Gonzales Montejo (Hospital Nacional Guillermo Almenara Irigoyen); Mariliadelcarmen Escalantesalas, Willy Alccaticona, Marvin Vargas, Georgechristian Manriquesila, Robinson Mas, Arazzelly del Pilar Paucar (Hospital Regional De Ayacucho); Armando Jose Roman Velasquez, Alina Robledo-Rabanal, Ludwing Alexander Zeta Solis, (Hospital III José Cayetano Heredia); Kenny Turpo Espinoza (Hospital Nacional Maria Auxiliadora); José Luis Hamasaki Hamaguchi, Erick Samuel Florez Farfan, Linda Alvi Madrid Barrientos, Juan Jaime Herrera Matta (Hospital De Policia);Philippines: John Jemuel V. Mora, Menold Archee P. Redota, Manuel Francisco Roxas, Maria Jesusa B. Mano, (The Medical City); Marie Dione Parreno-Sacdalan, Marie Carmela Lapitan, Christel Leanne Almanon (Department Of Surgery, Philippine General Hospital, University Of The Philippines Manila);Poland: Maciej Waledziak, Rafał Roszkowski, Michał Janik (Department Of General, Oncological, Metabolic And Thoracic Surgery, Military Institute Of Medicine, Warsaw); Anna Lasek, Piotr Major, Dorota Radkowiak, Mateusz Rubinkiewicz (2nd Department Of Surgery, Jagiellonian University Medical College);Portugal: Cristina Fernandes, Jose Costa-Maia, Renato Melo (Centro Hospitalar De Sao Joao);Romania: Liviu Muntean, Aurel Sandu Mironescu, Lucian Corneliu Vida (Spitalul Clinic De Copii Brasov); Amar Kourdouli, Mariuca Popa (Spital Judetean De Urgenta Din Craiova); Hogea Mircea (Spitalul Clinic Judetean Brasov); Mihaela Vartic, Bogdan Diaconescu, Matei Razvan Bratu, lonut Negoi, Mircea Beuran, Cezar Ciubotaru (Emergency Hospital of Bucharest);Rwanda: J.C Allen Ingabire, Mutabazi Alphonse, Norbert Uzabumwana (University Teaching Hospital of Kigali); Dieudonne Duhoranenayo (Kibungo Hospital);San Marino: Elio Jovine, Nicola Zanini, Giovanni Landolfo (San Marino State Hospital);Saudi Arabia: Murad Aljiffry, Faisal Idris, Mohammed Saleh A. Alghamdi, Ashraf Maghrabi, Abdulmalik Altaf, Eyad Khalifah, Ahmad Gudal, Adel Albiety, Aroub Alkaaki, Reham Alshareef, Ahmad Khoja, Abrar Nawawi, Mohammed Najjar, Sondos Turkustani, Sarah Sahel (Department of Surgery, Faculty of Medicine, King Abdulaziz University Hospital and Oncology Center, Jeddah) Ahmed Alzahrani, Ahmed Alghamdi, Wedyan Alhazmi, (King Fahad Hospital, Jeddah) Ghiath Al Saied, Mohammed Alamoudi, Muhammed Masood Riaz (King Fahad Medical City, Riyadh); Mazen Hassanain, Basmah Alhassan, Abdullah Altamimi, Reem Alyahya, Norah Alsubaie, Fatema Albastawis, Afnan Altamimi, Thamer Nouh, Roaa Khan, (King Khaled University Hospital);Serbia: Milan Radojkovic, Ljiljana Jeremic, Milica Nestorovic (Clinic For General Surgery, Clinical Center Nis);Singapore: Jia Hao Law, Keith Say Kwang Tan, Ryan Choon Kiat Tan, Joel Kin Tan, Lau Wen Liang Joel, Bettina Lieske, Xue Wei Chan, Faith Qi Hui Leong, Choon Seng Chong, Sharon Koh, Kai Yin Lee, Kuok Chung Lee (National University Hospital)South Africa: Kent Pluke, Britta Dedekind, Puyearashid Nashidengo, Mark Ian Hampton (Victoria Hospital Wynberg); Johanna Joosten, Sanju Sobnach, Liana Roodt, Anthony Sander, James Pape, Richard Spence (Groote Schuur); Niveshni Maistry (Charlotte Maxeke Johannesburg Academic Hospital); Phumudzo Ndwambi, Kamau Kinandu, Myint Tun (Leratong Hospital); Frederick Du Toit, Quinn Ellison, Sule Burger, DC Grobler, Lawrence Bongani Khulu (Tembisa Tertiary Provincial Hospital); Rachel Moore, Vicky Jennings, Astrid Leusink (Chris Hani Baragwanath Academic Hospital); Nazmie Kariem, Juan Gouws, Kathryn Chu, Heather Bougard, Fazlin Noor, Angela Dell (New Somerset Hospital); Sarah Rayne, Stephanie Van Straten, (Helen Joseph Hospital, University Of Witwatersrand); Arvin Khamajeet, Serge Kapenda Tshisola, Kalangu Kabongo (Stanger Hospital); Victor Kong (Edendale Hospital); Yoshan Moodley, Frank Anderson, Thandinkosi Madiba (Inkosi Albert Luthuli Central Hospital); Flip du Plooy (Mediclinic Potchefstroom); Leila Hartford, Gareth Chilton, Parveen Karjiker (Mitchell's Plain District Hospital); Matlouernest Mabitsela, Sibongileruth Ndlovu (Dr George Mukhari Academic Hospital); Maria Badicel, Robert Jaich (Milpark Hospital)Spain: Jaime Ruiz-Tovar 
(University Hospital Rey Juan Carlos); Luis Garcia-Florez, Jorge L. Otero-Diez, Virginia Ramosperez, Nuria Aguadosuarez (Hospital Universitario San Agustín); Javier Minguez Garcia, Sara Corral Moreno, Maria Vicenta Collado, Virginia Jimenez Carneros, Javier Garcia Septiem (Hospital Universitario de Getafe); Mariana Gonzalez, Antonio Picardo, Enrique Esteban, Esther Ferrero, Irene Ortega, (Infanta Sofía University Hospital); Eloy Espin-Basany, Ruth Blanco-Colino, Valeria Andriola (Hospital Valle De Hebron); Lorena i, Elisa Contreras, Carmen Garcíabernardo, Janet Pagnozzi, Sandra Sanz, Alberto Miyardeleon, Asnel Dorismé, Joseluis Rodicio, Aida Suarez, Jessica Stuva, Tamara Diazvico (Central University Hospital Of Asturias); Laura Fernandez-Vega, Carla Soldevila-Verdeguer, Fatima Sena-Ruiz, Natalia PujolCano, Paula Diaz-Jover, José Maria Garcia-Perez, Juan Jose Segura-Sampedro, Cristina Pineño-Flores, David Ambrona-Zafra, Andrea Craus-Miguel, Patricia Jimenez-Morillas, Angela Mazzella (Hospital Universitario Son Espases);Sri Lanka: A.B Jayathilake, S.P.B Thalgaspitiya, L.S.Wijayarathna, P.M.S.N.Wimalge (University Surgical Unit, Teaching Hospital Anuradhapura);St. Kitts And Nevis: Hakeem Ayomi Sanni, Aliyu Ndajiwo, Ogheneochuko Okenabirhie (Joseph N France Hospital)Sudan: Anmar Homeida, Abobaker Younis, Omer Abdelbagi Omer, Mustafa Abdulaziz, Ali Mussad, Ali M. Gilani (University of Gezira);Sweden: Yucel Cengiz, Ida Björklund, Sandra Ahlqvist, Sandra Ahlqvist, (Sundsvall Hospital); Anders Thorell, Fredrik Wogensen (Ersta Hospital); Arestis Sokratous, Michaela Breistrand (Mora Hospital); Hildur Thorarinsdottir (Helsingborgs Lasarett); Johanna Sigurdadottir, Maziar Nikberg, Abbas Chabok (Västmanlands Hospital Västerås); Maria Hjertberg (Department Of Surgery And Department Of Clinical And Experimental Medicine, Linköping University, Norrköping, Sweden); Peter Elbe, Deborah Saraste, Wiktor Rutkowski, Louise Forlin (Karolinska Universitetssjukhuset, Solna); Karoliina Niska, Malin Sund (Umea University Hospital)Switzerland: Dennis Oswald, Georgios Peros Rafael Bluelle, Katharina Reinisch, Daniel Frey, Adrian Palma (Gzo Spital Wetzikon); Dimitriaristotle Raptis, Lucius Zumbühl, Markus Zuber (Kantonsspital Olten); Roger Schmid, Gabriela Werder (Buergerspital Solothurn); Antonio Nocito, Alexandra Gerosa, Silke Mahanty (Kantonsspital Baden); Lukas Werner Widmer, Julia Müller, Alissa Gübeli (Hospital Davos); Grzegorz Zuk (Gzo Spital Wetzikon);Turkey: Osman Bilgin Gulcicek, Yuksel Altinel, Talar Vartanoglu, (Bagcilar Research And Training Hospital); Emin Kose, Servet Rustu Karahan, Mehmet Can Aydin (Okmeydanı Training And Research Hospital); Nuri Alper Sahbaz, Ilkay Halicioglu, Halil Alis (Bakirkoy Dr. Sadi Konuk Training And Research Hospital); Ipek Sapci, Can Adıyaman, Ahmet Murat Pektaş, Turgut Bora Cengiz, Illkan Tansoker, Vedatcan Ișler, Muazzez Cevik, Deniz Mutlu, Volkan Ozben, Berk Baris Ozmen, Sefa Bayram, Sinem Yolcu, Berna Buse Kobal, Ömer Faruk Toto, Haluk Cem Çakaloğlu (Acibadem University School of Medicine, Atakent Hospital); Kagan Karabulut, Vahit Mutlu, Bahar Busra Ozkan (Ondokuz Mayis University Medical Faculty); Saban Celik, Anil Semiz, Selim Bodur, Enisburak Gül, Busra Murutoglu, Reyyan Yildirim, Bahadir Emre Baki, Ekin Arslan, Mehmet Ulusahin, Ali Guner (Karadeniz Technical University Faculty Of Medicine);United Kingdom: Nathan Walker, Nikhita Shrimanker, Michael Stoddart, Simon Cole (Royal United Hospital Bath); Ryan Breslin, Ravi Srinivasan (Blackpool Victoria Hospital); Mohamed Elshaer, Kristina Hunter, Ahmed Al-Bahrani (Watford General Hospital); Ignatius Liew, Nora Grace Mairs, Alistair Rocke, Lachlan Dick, Mobeen Qureshi (Inverclyde Royal Hospital); Debkumar Chowdhury (University Hospital Ayr); Naomi Wright, Clare Skerritt, (Guy's And St. Thomas Hospitals); Adrienne Ho, Tharindra Dissanayake, Athula Tennakoon, Wadah Ali, (Pilgrim Hospital, United Lincolnshire Hospitals NHS Trust); Shujing Jane Lim, Charlene Tan, Stephen 0 Neill, Catrin Jones (Victoria Hospital Kirkcaldy); Stephen Knight, Dima Nassif, Abhishek Sharma (Perth Royal Infirmary); Oliver Warren, Rebecca White, Aia Mehdi, Nathan Post, Eliana Kalakouti, Enkhbat Dashnyam, Frederick Stourton (Chelsea And Westminster Hospital); loannis Mykoniatis, Chelise Currow, (Northampton General Hospital); Francisca Wong, Ashish Gupta, Veeranna Shatkar (Queen s Hospital, BHR University Hospitals NHS Trust); Joshua Luck, Suraj Kadiwar, Alexander Smedley (North Middlesex University Hospital); Rebecca Wakefield, Philip Herrod, James Blackwell, Jonathan Lund, (Royal Derby Hospital); Fraser Cohen, Ashwath Bandi, Stefano Giuliani (St Georges Hospital); Giles BondSmith, Theodore Pezas, Neda Farhangmehr, Tomas Urbonas, Miklos Perenyei (John Radcliffe Hospital, Oxford); Philip Ireland, Natalie Blencowe, Kirk Bowling, David Bunting (Gloucestershire Royal Hospital); Lydia Longstaff, Neil Smart, Kenneth Keogh (Royal Devon \& Exeter Hospital); Hyunjin Jeon, Muhammad Rafaih lqbal, Shivun Khosla, Anna Jeffery, James Perera (Maidstone \& Tunbridge Wells NHS Trust); Ella Teasdale (Western Isles Hospital); Ahmad Aboelkassem Ibrahem, Tariq Alhammali, Yahya Salama (Kettering General Hospital NHS Trust); Rakan Kabariti, Shaun Oram (Nevill Hall Hospital); Thomas Kidd, Fraser Cullen, Christopher Owen, Michael Wilson, Seehui Chiu, Hannah Sarafilovic, (Ninewells Hospital); Jennifer Ploski, Elizabeth Evans, Athar Abbas, Sylvia Kamya, Norzawani Ishak, Carly Bisset, Cedar Andress, Ye Ru Chin (Royal Alexandra Hospital, Paisley); Priya Patel, David Evans (University Hospital, Wales); Anna Jeffery, James Perera (Maistone and Tunbridge Wells NHS Trust); Aidan Haslegrave, Adam Boggon, Kirsten Laurie, Katie
Connor, Thomas Mann (Borders General Hospital); Dmitri Nepogodiev, Anahita Mansuri, Rachel Davies, Ewen Griffiths (University Hospitals Birmingham NHS Trust); Aized Raza Shahbaz, Calvin Eng, Farhat Din, Ariadne L heveder, Esther H.G. Park, Ramanish Ravishankar, Kirsten Mcintosh, Jih Dar Yau, Luke Chan, Susan Mcgarvie (Western General Hospital, Edinburgh); Lingshan Tang, Hui Lim, Suhhuey Yap, Jay Park, Zhanherr Ng, Shahrukh Mirza, Yun Lin Ang, Luke Walls, Ella Teasdale, Chloe Roy, Simon Paterson-Brown, Julian Camilleri-Brennan, Kenneth Mclean, Michelle D souza, Savva Pronin, David Ewart Henshall, Eunicezuling Ter (Royal Infirmary Of Edinburgh); Dina Fouad, Ashish Minocha (Norfolk And Norwich University Hospital); William English, Catrin Morgan, Dominic Townsend, Laura Maciejec, Shareef Mahdi, Onyinye Akpenyi, Elisabeth Hall, Hanaan Caydiid, Zakaria Rob, Tom Abbott, Hew D Torrance (The Royal London Hospital); Gareth Irwin, Robin Johnston (Ulster Hospital Dundonald); Mohammedakil Gani, Gianpiero Gravante (Leicester Royal Infirmary); Shivanchan Rajmohan, Kiran Majid, Shiva Dindyal, Christopher Smith (West Middlesex University Hospital); Madanmohan Palliyil, Sanjay Patel, Luke Nicholson, Neil Harvey, Katie Baillie, Sam Shillito, Suzanne Kershaw, Rebecca Bamford, Peter Orton (Stockport NHS Foundation Trust); Elke Reunis, Robert Tyler, Waicheong Soon (Good Hope Hospital); Guled M. Jama, Dharminder Dhillon, Khyati Patel (Walsall Manor Hospital, Walsall); Shayanthan Nanthakumaran, Rachel Heard, Kar Yan Chen (Aberdeen Royal Infirmary); Behrad Barmayehvar, Uttaran Datta, Sivesh K Kamarajah (Heartlands Hospital); Sobhana Iftekhartani (Nottingham University Hospital NHS Trust); Eimear Monaghan, Philippa Donnelly, Michael Walker (Raigmore Hospital); Jehangirshaw Parakh, Sarah Blacker, Anil Kaul (Whiston Hospital); Arjun Paramasivan (Darlington Memorial Hospital); Sameh Farag, Ashrafun Nessa, Salwa Awadallah (Worthing Hospital, Western Sussex Hospital NHS Foundation Trust); Jieqi Lim, James Cheankhunng (Queen Elizabeth University Hospital, Glasgow);United States: Katherine Gash, Ravi P. Kiran, Alice Murray (New York Presbyterian Hospital / Columbia University Medical Center); Eric Etchill, Mohini Dasari, Juan Puyana (University Of Pittsburgh Medical Center - Presbyterian); Nadeem Haddad, Martin Zielinski, Asad Choudhry (Mayo Clinic); Celeste Caliman, Mieshia Beamon, Therese Duane (John Peter Smith Hospital); Ragavan Narayanan, Mamta Swaroop (Northwestern Memorial Hospital / Northwestern University); Jonathan Myers, Rebecca Deal, Erik Schadde (Rush University Medical Centre); Mark Hemmila, Lena Napolitano, Kathleen To (University Of Michigan Medical Center)Zambia: Alex Makupe, Joseph Musowoya, Mayaba Maimbo (Ndola Central Hospital); Niels Van Der Naald, Dayson Kumwenda, Alex Reece-Smith, Kars Otten, Anna Verbeek, Marloes Prins (St Francis Mission Hospital) Data Validators:Argentina: Alibeth Andres Baquero Suarez (Simplemente Evita), Ruben Balmaceda (Hospital Lagomaggiore);Barbados: Chelsea Deane (Queen Elizabeth Hospital);Croatia: Emilio Dijan (Zadar General Hospital);Egypt: Mahmoud Elfiky (Kasr Al Ainy Faculty of Medicine, Cairo University);Finland: Laura Koskenvuo (Helsinki University Hospital);France: Aurore Thollot (CHU Poitiers), Bernard Limoges (CHU Limoges), Carmen Capito (Hopital Necker Enfants Malades, APHP), Challine Alexandre (Hopital Cochin, APHP), Henri Kotobi (Trousseau Hospital, APHP), Julien Leroux (CHU Rouen), Julien Rod (CHU Caen), Kalitha Pinnagoda (CHU Toulouse), Nicolas Henric (CHU Angers), Olivier Azzis (CHU Rennes), Olivier Rosello (CHU Nice), Poddevin Francois (GHICL), Sara Etienne (CHU Saint Etienne); Philippe Buisson (CHU Amiens Picardie), Sophian Hmila (Hopital Robert Ballanger, Paris);Ghana: Joe-Nat Clegg-Lamptey (Korle Bu Teaching Hospital), Osman Imoro (Baptist Medical Centre), Owusu Emmanuel Abem (Komfo Anokye Teaching Hospital), Paul Wondoh (Upper West Regional Hospital);Greece: Dimitrios Papageorgiou (Naval And Veterans Hospital Of Athens), Vasiliki Soulou (Anticancer Hospital Of Athens Agios Savvas);Guatemala: Sabrina Asturias (Hospital Herrera Llerandi Amedesgua), Lenin Peña (Hospital General San Juan De Dios);India: Basant Kumar (Sanjay Gandhi Post Graduate Institute Of Medical College Lucknow); Ireland: Donal B 0 Connor (Tallaght Hospital, Trinity College Dublin);Italy: Alberto Realis Luc (Santa Rita Clinic, Vercelli), Alfio Alessandro Russo (Treviglio Hospital), Andrea Ruzzenente (Azienda Ospedaliera Universitaria Integrata di Verona), Antonio Taddei (Azienda Ospedaliera Universitaria Careggi), Camilla Cona (IOV - Istituto Oncologico Veneto), Corrado Bottini (Sant Antonio Abate Hospital, Gallarate), Giovanni Pascale (Azienda Ospedaliero-Universitaria di Ferrara), Giuseppe Rotunno (Nicola Giannettasio Hospital, Rossano), Leonardo Solaini (University Of Brescia, Spedali Civili Di Brescia), Marco Maria Pascale (Fondazione Policlinico Universitario 'Agostino Gemelli'), Margherita Notarnicola (University Of Bari 'Aldo Moro'), Mario Corbellino (Ospedale Luigi Sacco Milano), Michele Sacco (Federico II University of Naples), Paolo Ubiali (Azienda per L'Assistenza Sanitaria N. 5 'Friuli Occidentale', Pordenone), Roberto Cautiero (Second University of Naples), Tommaso Bocchetti, (Sant'Andrea Hospital, Sapienza University of Rome), Elena Muzio, (S. Andrea Hospital, Poll-Asl 5, La Spezia); Vania Guglielmo (Policlinico Umberto I, Emergency Surgery Department); Eugenio Morandi (Ospedale di Rho - ASST Rhodense), Patrizio Mao (San Luigi Gonzaga Hospital, Orbassano); Emilia De Luca (Department of Medical and Surgical Sciences, Policlinico Universitario Mater Domini Campus 
Salvatore Venuta, Catanzaro).Jordan: Farah Mahmoud Ali (Jordan University Hospital);Lithuania: Justas Žilinskas (Klaipeda Republic), Kestutis Strupas (Vilnius University Hospital), Paulius Kondrotas (Taurage County Hospital), Robertas Baltrunas (Rokiskis District Municipality Hospital); Juozas Kutkevicius (Department Of General Surgery, Lithuanian University Of Health Sciences), Povilas Ignatavicius (Hospital Of Lithuanian University Of Health Sciences Kaunas Clinics);Malaysia: Choy Ling Tan (Hospital Sultanah Aminah), Jia Yng Siaw (Hospital Sibu), Sir Young Yam (Penang Medical College); Ling Wilson (Sarawak General Hospital), Mohamed Rezal Abdul Aziz (University Malaya Medical Centre);Malta: John Bondin (Mater Dei Hospital);Mexico: Carmina Diaz Zorrilla (Hospital Espanol De Veracruz);Morocco: Anass Majbar (Centre Hospitalier Ibn Sina Rabat);Nigeria: Danjuma Sale (Barau Dikko Teaching Hospital), Lawal Abdullahi (Kano Aminu), Olabisi Osagie (University Of Abuja Teaching Hospital), Omolara Faboya (Lagos Lasuth); Adedeji Fatuga (Lagos Luth), Agboola Taiwo (Babcock University Teaching Hospital), Emeka Nwabuoku (Ahmadu Bello University Teaching Hospital);Norway: Marte Bliksøen (0slo University Hospital);Pakistan: Zain Ali Khan (Bahawal Victoria Hospital, Bahawalpur);Paraguay: Jazmin Coronel (Hospital de Clínicas, II Cátedra de Clínica Quirúrgica, Universidad Nacional de Asunción)Peru: Cesar Miranda (Hospital Nacional Cayetano Heredia), Idelso Vasquez (Lima Almenara), Luis M. HelgueroSantin (Hospital Regional III Jose Cayetano Heredia - Piura);Rwanda: Jennifer Rickard (Centre Hospitalier Universitaire De Kigali);Romania: Aurel Mironescu (Spitalul Clinic De Copii Brasov);Saint Kitts and Nevis: Adesina Adedeji (Joseph N France Hospital);Saudi Arabia: Saleh Alqahtani (King Fahad General Hospital);South Africa: Max Rath (Groote Schuur Hospital), Michael Van Niekerk (New Somerset Hospital), Modise Zacharia Koto (Dr George Mukhari Academic Hospital); Roel Matos-Puig (Stanger Hospital);Sweden: Leif Israelsson (Sundsvall);Switzerland: Tobias Schuetz (Kantonsspital Olten);Turkey: Mahmut Arif Yuksek (Ondokuz Mayis University), Meric Mericliler (Acibadem University School of Medicine, Atakent Hospital), Mehmet Uluşahin (Karadeniz Technical University Farabi Hospital);United Kingdom: Bernhard Wolf (Raigmore Hospital Inverness), Cameron Fairfield (Royal Infirmary Of Edinburgh), Guo Liang Yong (Perth Royal Infirmary), Katharine Whitehurst (Royal Devon And Exeter), Michael Wilson (Ninewells Hospital And Medical School), Natalie Redgrave (John Radcliff Hospital, Oxford); Caroluce K Musyoka (Royal Alexandra Hospital), James Olivier (Royal United Hospital Bath), Kathryn Lee (Queen Elizabeth Birmingham), Michael Cox (Royal Derby Hospital), Muhamed M H Farhan-Alanie (Inverclyde Royal Hospital), Rory Callan (North Middlesex University Hospital)Zambia: Chali Chibuye (Ndola Central Hospital) Protocol Translators:Arabic, Tebian Hassanein Ahmed Ali, Syrine Rekhis, Muna Rommaneh, Oday Halhouli;Chinese, Zi Hao Sam;French, Ismail Lawani;Greek, Vasileios Kalles;|talian, Francesco Pata, Gabriela Elisa Nita, Federico Coccolini, Luca Ansaloni;Portuguese, Thays Brunelli Pugliesi, Gabriel Pardo;Spanish, Ruth Blanco

Contributors Listed members of the Steering group were responsible for the inception and design of this study, including design of the data collection instruments and critical review of the protocol and its translation. The listed national and local authors organised recruitment, coordinated site approvals and collected data. The writing group was responsible for the statistical analysis, interpretation of data, drafting and revision of the manuscript. Authors are listed under their responsibilities in the 'Authorship' section of this manuscript.

Funding This study is funded by DFID-MRC-Wellcome Trust Joint Global Health Trial Development Grant (MR/N022114/1). A National Institute of Health Research (NIHR) Global Health Research Unit Grant (NIHR 17-0799) is supporting the establishment of surgical research units in a subset of contributing low-income countries. The views expressed are those of the authors and not necessarily those of the NHS, the NIHR or the UK Department of Health and Social Care.

Competing interests JEF reports personal fees from KPMG Global Healthcare Practice outside the submitted work. The authors have no other conflicts of interest relevant to this article to disclose.

\section{Patient consent for publication Not required}

Ethics approval A UK National Health Service Research Ethics review considered this study exempt from formal research registration (South East Scotland Research Ethics Service, reference NR/1510AB5). Individual centres were responsible for obtaining their own audit or institutional approval as per local guidelines, and ethical approval was obtained in countries where local research ethics committees deemed it a requirement.

Provenance and peer review Not commissioned; externally peer reviewed.

Data availability statement Data are available upon request. Deidentified individual participant data (including data dictionaries) will be made available, in addition to study protocols, the statistical analysis plan, and the informed consent form. The data will be made available upon publication to researchers who provide a methodologically sound proposal for use in achieving the goals of the approved proposal. Proposals should be submitted to ewen.harrison@ed.ac.uk. Data can be explored using an interactive web application at http://ssi.globalsurg.org.

Supplemental material This content has been supplied by the author(s). It has not been vetted by BMJ Publishing Group Limited (BMJ) and may not have been peer-reviewed. Any opinions or recommendations discussed are solely those of the author(s) and are not endorsed by BMJ. BMJ disclaims all liability and responsibility arising from any reliance placed on the content. Where the content includes any translated material, BMJ does not warrant the accuracy and reliability of the translations (including but not limited to local regulations, clinical guidelines, terminology, drug names and drug dosages), and is not responsible for any error and/or omissions arising from translation and adaptation or otherwise.

Open access This is an open access article distributed in accordance with the Creative Commons Attribution 4.0 Unported (CC BY 4.0) license, which permits others to copy, redistribute, remix, transform and build upon this work for any purpose, provided the original work is properly cited, a link to the licence is given, and indication of whether changes were made. See: https://creativecommons.org/ licenses/by/4.0/

\section{REFERENCES}

1 Horwitz JR, Chwals WJ, Doski JJ, et al. Pediatric wound infections: a prospective multicenter study. Ann Surg 1998;227:553-8.

2 Shrime MG, Dare A, Alkire BC, et al. A global country-level comparison of the financial burden of surgery. Br J Surg 2016;103:1453-61.

3 Khoshbin A, So JP, Aleem IS, et al. Antibiotic prophylaxis to prevent surgical site infections in children: a prospective cohort study. Ann Surg 2015;262:397-402.

4 Uludag O, Rieu P, Niessen M, et al. Incidence of surgical site infections in pediatric patients: a 3-month prospective study in an academic pediatric surgical unit. Pediatr Surg Int 2000;16:417-20.

5 Davenport M, Doig CM. Wound infection in pediatric surgery: a study in 1,094 neonates. J Pediatr Surg 1993;28:26-30.

6 Allegranzi B, Bagheri Nejad S, Combescure C, et al. Burden of endemic health-care-associated infection in developing countries: systematic review and meta-analysis. Lancet 2011;377:228-41.

7 Bagheri Nejad S, Allegranzi B, Syed SB, et al. Health-careassociated infection in Africa: a systematic review. Bull World Health Organ 2011;89:757-65.

8 GlobalSurg Collaborative. Determining the worldwide epidemiology of surgical site infections after gastrointestinal resection surgery: protocol for a multicentre, international, prospective cohort study (GlobalSurg 2). BMJ Open 2017;7:e012150.

9 Bhangu A, Kolias AG, Pinkney T, et al. Surgical research collaboratives in the UK. Lancet 2013;382:1091-2.

10 GlobalSurg Collaborative. Surgical site infection after gastrointestinal surgery in high-income, middle-income, and low-income countries: a prospective, international, multicentre cohort study. Lancet Infect Dis 2018;18:516-25

11 Lang TA, Altman DG. Basic statistical reporting for articles published in biomedical journals: the "Statistical Analyses and Methods in the Published Literature" or the SAMPL Guidelines. Int J Nurs Stud 2015;52:5-9.

12 von Elm E, Altman DG, Egger M, et al. The strengthening the reporting of observational studies in epidemiology (STROBE) statement: guidelines for reporting observational studies. PLoS Med 2007;4:e296.

13 Harris PA, Taylor R, Thielke R, et al. Research electronic data capture (REDCap)--a metadata-driven methodology and workflow process for providing translational research informatics support. J Biomed Inform 2009;42:377-81.

14 Centers for Disease Control and Prevention. Site infection (SSI). $C D C$, 2018: 1-32. https://www.google.co.uk/url?sa=t\&rct=j\&q=\& esrc $=s \&$ source $=$ web\& $c d=3 \& c a d=r j a \& u a c t=8 \&$ ved $=0$ ahUKEwiEtPD QOrLYAhWKY1AKHQUqAvlQFgg3MAI\&url=https\%3A\%2F\%2Fwww. cdc.gov\%2Fnhsn\%2Fpdfs\%2Fpscmanual\%2F9pscssicurrent.pdf\& usg=AOvVaw1pfxZuFL-VI2tIEfXGHiDP

15 Allegranzi B, Pittet D. Healthcare-Associated infection in developing countries: simple solutions to meet complex challenges. Infect Control Hosp Epidemiol 2007;28:1323-7.

16 Shears P. Poverty and infection in the developing world: healthcarerelated infections and infection control in the tropics. J Hosp Infect 2007;67:217-24.

17 Ombelet S, Ronat J-B, Walsh T, et al. Clinical bacteriology in low-resource settings: today's solutions. Lancet Infect Dis 2018;18:e248-58. 
18 Li X, Zhang J, Sang L, et al. Laparoscopic vs conventional appendectomy - A meta-analysis of randomised controlled trials. Langenbeck's Arch Surg 2010;383:289-95.

19 GlobalSurg Collaborative. Laparoscopy in management of appendicitis in high-, middle-, and low-income countries: a multicenter, prospective, cohort study. Surg Endosc 2018;32:3450-66.

20 Chao TE, Mandigo M, Opoku-Anane J, et al. Systematic review of laparoscopic surgery in low- and middle-income countries: benefits, challenges, and strategies. Surg Endosc 2016;30:1-10.

21 Sitkin NA, Farmer DL. Congenital anomalies in the context of global surgery. Semin Pediatr Surg 2016;25:15-18.

22 Sitkin NA, Ozgediz D, Donkor P, et al. Congenital anomalies in lowand middle-income countries: the unborn child of global surgery. World J Surg 2015;39:36-40.
23 Liu L, Oza S, Hogan D, et al. Global, regional, and national causes of child mortality in 2000-13, with projections to inform post-2015 priorities: an updated systematic analysis. Lancet 2015;385:430-40.

24 Global Burden of Disease Pediatrics Collaboration, Kyu HH, Pinho $\mathrm{C}$, et al. Global and national burden of diseases and injuries among children and adolescents between 1990 and 2013: findings from the global burden of disease 2013 study. JAMA Pediatr 2016;170:267-87.

25 Nepogodiev D, Martin J, Biccard B, et al. Global burden of postoperative death. Lancet 2019;393:401.

26 Allegranzi B, Bischoff P, de Jonge S, et al. New who recommendations on preoperative measures for surgical site infection prevention: an evidence-based global perspective. Lancet Infect Dis 2016;16:e276-87.

27 Aiken AM, Karuri DM, Wanyoro AK, et al. Interventional studies for preventing surgical site infections in sub-Saharan Africa - A systematic review. Int J Surg 2012;10:242-9. 